\title{
Asymptotic analysis of wall modes in a flexible tube revisited
}

\author{
V. Shankar and V. Kumaran ${ }^{\mathrm{a}}$ \\ Department of Chemical Engineering, Indian Institute of Science, Bangalore 560 012, India
}

Received 12 June 2000 and Received in final form 8 November 2000

\begin{abstract}
The stability of wall modes in fluid flow through a flexible tube of radius $R$ surrounded by a viscoelastic material in the region $R<r<H R$ is analysed using a combination of asymptotic and numerical methods. The fluid is Newtonian, while the flexible wall is modelled as an incompressible viscoelastic solid. In the limit of high Reynolds number $(R e)$, the vorticity of the wall modes is confined to a region of thickness $O\left(R e^{-1 / 3}\right)$ in the fluid near the wall of the tube. Previous numerical studies on the stability of Hagen-Poiseuille flow in a flexible tube to axisymmetric disturbances have shown that the flow could be unstable in the limit of high Re, while previous high Reynolds number asymptotic analyses have revealed only stable modes. To resolve this discrepancy, the present work re-examines the asymptotic analysis of wall modes in a flexible tube using a new set of scaling assumptions. It is shown that wall modes in Hagen-Poiseuille flow in a flexible tube are indeed unstable in the limit of high $R e$ in the scaling regime $\operatorname{Re} \sim \Sigma^{3 / 4}$. Here $\Sigma$ is a nondimensional parameter characterising the elasticity of the wall, and $\Sigma \equiv \rho G R^{2} / \eta^{2}$, where $\rho$ and $\eta$ are the density and viscosity of the fluid, and $G$ is the shear modulus of the wall medium. The results from the present asymptotic analysis are in excellent agreement with the previous numerical results. Importantly, the present work shows that the different types of unstable modes at high Reynolds number reported in previous numerical studies are qualitatively the same: they all belong to the class of unstable wall modes predicted in this paper.
\end{abstract}

PACS. 83.50.-v Deformation; material flow - 47.15.Fe Stability of laminar flows $-47.60 .+\mathrm{i}$ Flows in ducts, channels, nozzles and conduits

\section{Introduction}

The flow of fluid through flexible-walled tubes and channels is a prototypical situation encountered in many biological systems and in some biotechnological applications. The flow of blood and other fluids in the body takes place through flexible tubes, and the separation and purification processes in bio-technological processes often involve flow in tubes and channels made up of polymer matrices and membranes. Traditionally, these flows have been analysed using models similar to those for the flow in a rigid tube. However, the dynamics of fluid flow past flexible solid surfaces is qualitatively different from that of rigid surfaces because of the coupling between the fluid and wall dynamics, and the elasticity of the surface could affect the fluid flow. In particular, this coupling could influence the transition from laminar to turbulent flow in such systems, and this has indeed been observed in earlier experiments. Experiments conducted by Krindel and Silberberg [1] in a gel-walled tube indicate that there is an anomalous drag force at a Reynolds numbers $(R e)$ as low as about 600 , and the authors concluded that this is due to a transition to a turbulent flow at a Reynolds number which is far lower than the critical Reynolds number for the flow through a

\footnotetext{
a e-mail: kumaran@chemeng.iisc.ernet.in
}

rigid tube (around 2100). The transition $R e$ was found to depend on the elasticity of the wall in addition to the fluid properties indicating that the wall dynamics plays a significant role in the transition events. Motivated by these experimental results, there has been a renewed interest in the recent years in the the understanding of the stability of fluid flow through flexible tubes and channels [2-7]. The salient results of these studies that are pertinent to the present work are summarised below.

1. The Hagen-Poiseuille flow in a flexible tube could become unstable even in the limit of zero Reynolds number [2] when the dimensionless velocity $(V \eta / G R)$ increases beyond a critical value. Here, $V$ is the maximum velocity in the tube, $\eta$ is the fluid viscosity, $G$ is the modulus of elasticity of the wall material and $R$ is the tube radius. The instability is driven by the transport of energy from the mean flow to the fluctuations due to the deformation work done by the mean flow at the flexible surface. This class of modes are referred to as 'viscous modes'. A similar instability was predicted for the case of Couette flow past a flexible surface by Kumaran, Fredrickson and Pincus [8].

2. The existence of unstable modes even in the low $R e$ limit in the case of fluid flow past soft solid surfaces was recently confirmed experimentally by Kumaran and Muralikrishnan [7]. The critical velocity required for 
initiating the instability in the experiments was found to be in good agreement with the theoretical predictions of [8], with no adjustable parameters, for a wide range of gel thicknesses and elastic moduli. These new experiments have provided convincing evidence for the viscous instability predicted in [2] and [8].

3. The stability of Hagen-Poiseuille flow in a flexible tube was analysed in the limit of high $R e$ in [3]. In this limit, the flow is inviscid in the core of the tube, and there is a wall layer of thickness $O\left(R e^{-1 / 2}\right)$ smaller than the tube radius where the viscous stresses are $O\left(R e^{-1 / 2}\right)$ smaller than the inertial stresses. An asymptotic analysis in the small parameter $R e^{-1}$ was used, and in the leading approximation the real part of the growth rate is zero, indicating that the perturbations are neutrally stable at this level of approximation. The $O\left(R e^{-1 / 2}\right)$ correction to the growth rate due to the viscous stresses in the wall layer turns out to be negative, indicating that the flow is stable in the limit of high $R e$. These modes are referred to as 'inviscid modes'.

4. The low Reynolds number analysis $[2,8]$ showed that the fluid flow could become unstable when the dimensionless velocity is increased beyond a critical value, but the high Reynolds number analysis [3] indicated that the inviscid modes are always stable. The above asymptotic results are rather paradoxical, because they seem to suggest that the flow in the low Reynolds number regime could become unstable, while the flow in the high Reynolds number regime is always stable. In order to resolve this paradox, a numerical continuation of the unstable viscous modes into the intermediate Reynolds number regime was undertaken in [4] for the case of Couette flow in a flexible channel, and in [5] for the case of Hagen-Poiseuille flow in a flexible tube. In these studies, it was found that the low $R e$ instability does persist into the intermediate and high Reynolds number regime, but the critical Reynolds number is much larger than the Reynolds number at which the inviscid modes are observed for a given set of fluid and wall properties. In addition, a boundary layer of thickness $O\left(R e^{-1 / 3}\right)$ smaller than the width of the channel thickness or tube radius was observed for these unstable modes in the high Reynolds number regime. This is very different from the boundary layer of thickness $O\left(R e^{-1 / 2}\right)$ for the inviscid modes. The numerical results of both these studies showed that the critical Reynolds number in this case scales as $R e \propto \Sigma^{\alpha}$, where the exponent $\alpha$ is between 0.7 and 0.75 . Here, the parameter $\Sigma=\left(\rho G R^{2} / \eta^{2}\right)$, where $\rho$ is the fluid density. The $O\left(R e^{-1 / 3}\right)$ scaling is characteristic of a set of modes called 'wall modes'.

There has been considerable work done on the instability leading to the collapse of a flexible tube due to the difference between the internal and external pressures (see, for example $[9,10])$. This has physiological relevance, since similar flows are encountered in air flow through respiratory passages. In these studies, the flow is in the turbulent regime, and the cross-sectional area of the tube is related to the difference between the internal and external pressures. The present analysis is qualitatively different from these studies, because the basic flow is laminar, and the instability of the laminar flow in a tube with viscoelastic walls is examined. The instability leads to oscillations of the walls and a modification of the flow, but does not result in a significant change in the tube geometry. There have been some other studies which have focussed on the numerical computation of fluid flow in a tube with an elastic membrane insertion. Pedrizzetti [11] numerically computed the unsteady flow in a circular tube with a finite length elastic membrane in the otherwise rigid duct, using the theory of finite elasticity for the membrane. This numerical study showed that when a steady fluid discharge is imposed on the downstream rigid duct, the fluid-wall interaction develops travelling waves along the membrane whose period depends on the membrane elasticity. The presence of viscous stresses in the membrane was found to stabilise the instability. The author concluded that the stationary oscillations observed in the computations could be related to the 'divergence' instability in boundary layer flow past compliant walls (see, for example [12]).

The present study addresses the stability of wall modes in a flexible tube using a combination of asymptotic and numerical methods. Wall modes are a class of solutions in the high Reynolds number limit where the vorticity in the fluid is confined to a very thin layer near the wall of the tube (referred henceforth as the 'wall layer') of thickness $O\left(R e^{-1 / 3}\right)$ smaller than the radius of the tube. The damping rate of these modes is $O\left(R e^{-1 / 3}\right)$ smaller than the strain rate in the fluid. These modes are distinct from the inviscid modes, because in the case of wall modes the velocity components in the inviscid flow in the bulk of the tube are small compared to the tangential velocity in the wall layer. These modes were first studied in [13] and [14] for the case of Hagen-Poiseuille flow in a rigid tube, and these asymptotic studies showed that wall modes are always stable in a rigid tube. However, these studies also showed that the wall modes are the least stable modes in a rigid tube, and so it is important to study the stability characteristics of the wall modes in flow through a flexible tube. Since the vorticity in the fluid is confined near the wall of the tube, the elasticity of the wall can affect the stability of the wall modes in the case of fluid flow through flexible tubes.

The stability of wall modes in a flexible tube was analysed using an asymptotic analysis in the high Reynolds number limit by Kumaran [15]. This analysis mainly focussed on the regime $R e \gg 1$, and $\Lambda \equiv \operatorname{Re}^{1 / 3}\left(G / \rho V^{2}\right) \sim$ 1. Here, $R e \equiv R V \rho / \eta$ is the Reynolds number, $\rho, \eta$ are respectively the density and viscosity of the Newtonian fluid, $V$ is the maximum velocity of the Hagen - Poiseuille flow, $G$ is the shear modulus of the wall material, and $R$ is the radius of the flexible tube. A scaling analysis showed that the elastic stresses in the wall and the normal stresses in the fluid were balanced in the limit $\Lambda \sim 1$. In the wall layer, the inertial and viscous fluid stresses are of the same magnitude. However, in the wall material the elastic stress are large compared to the inertial 
stresses in the limit $\Lambda \sim 1$. An asymptotic analysis in the small parameter $R e^{-1 / 3}$ was used to determine the growth rate, which showed that there are multiple solutions to the growth rate. In the limit $\Lambda \rightarrow \infty$, which corresponds to elastic stresses in the wall very large compared to viscous stresses in the fluid (i.e. the rigid tube limit), the solutions to the growth rate converged to the solutions of Gill [14] for the case of wall modes in a rigid tube. In the opposite limit of $\Lambda \rightarrow 0$, which corresponds to a wall with very small elasticity, the growth rates were again found to be stable. The transition from $\Lambda \gg 1$ to $\Lambda \ll 1$ was found to be smooth. However, there is one mode in the limit $\Lambda \ll 1$ in a flexible tube whose growth rate does not converge to any of the rigid tube modes, but the frequency of this mode diverges as $\Lambda$ in the limit of a rigid tube $(\Lambda \rightarrow \infty)$, and the decay rate was found to decrease as $\Lambda^{-1 / 2}$ in the limit $\Lambda \rightarrow \infty$. It was then concluded in [15] that this represents the least stable wall mode in a flexible tube. An increase in wall flexibility (i.e. a decrease in the elasticity of the wall medium) has a stabilising effect on this mode.

This least stable wall mode in a flexible tube, which is absent in the case of a rigid tube, was continued numerically to the $\Lambda \ll 1$ regime in [6]. That study showed that this particular mode becomes unstable when $\Lambda$ was decreased below a transition value at a given $R e$, with $R e$ ranging between 1000 and 10000 . The neutral stability curves for this unstable mode was obtained using a numerical continuation scheme, and the Reynolds number for which there is a cross-over from stable to unstable modes was determined as a function of the parameter $\Sigma$. The parameter $\Sigma$, defined as $\Sigma \equiv \rho G R^{2} / \eta^{2}$, is a flowindependent quantity which is proportional to the shear modulus $G$ of the flexible tube. The numerical results revealed that the $R e$ for neutral modes decreases proportional to $\Sigma^{1 / 2}$ in the limit $\Sigma \ll 1$, and shows rather complex behaviour in the intermediate regime. In the limit $\Sigma \gg 1$, the Reynolds number at which there is a transition from stable to unstable modes increases proportional to $\Sigma^{\alpha}$, where $\alpha$ was found to be between 0.7 and 0.75 . Interestingly, the numerical continuation of 'viscous modes' to the intermediate $R e$ regime in [5] for the case of HagenPoiseuille flow in a flexible tube and in [4] for the case of Couette flow past a flexible surface, also showed that the Reynolds number for neutral modes scales as $R e \sim \Sigma^{\alpha}$ for $\Sigma \gg 1$, where $\alpha$ was again found to be between 0.7 to 0.75 . In addition, in both these studies, the numerical eigenfunctions showed the existence of a boundary layer of thickness of $O\left(R e^{-1 / 3}\right)$ in the limit $R e \gg 1$.

The above discussion illustrates that the previous numerical studies on the stability of Hagen-Poiseuille flow in a flexible tube show the presence of unstable modes even in the limit of high $R e$, with $R e \sim \Sigma^{3 / 4}$. This behaviour is characteristic of both 'intermediate Reynolds number modes' $[4,5]$ and 'wall modes' [6], and both these modes were characterised by the presence of a boundary layer of thickness of $O\left(R e^{-1 / 3}\right)$ near the wall of the tube where viscous effects are dominant. However, the earlier high $R e$ asymptotic analysis of wall modes in a flexible tube [15], which included this $O\left(R e^{-1 / 3}\right)$ viscous wall layer, pre- dicted only stable modes. It is important to note that the earlier asymptotic analysis was restricted to the regime $R e^{1 / 3}\left(G / \rho V^{2}\right) \sim 1$, which implies that $R e \sim \Sigma^{3 / 5}$. In addition, the other high $R e$ asymptotic analysis [3], which probed the regime $R e \sim \Sigma^{1 / 2}$ (i.e. the inviscid modes), showed that the flow is stable in the limit of high Reynolds number. However, the numerical studies [4-6] showed the existence of unstable modes in the scaling regime more close to $R e \sim \Sigma^{3 / 4}$ for $\Sigma \gg 1$.

The previous numerical results therefore raise the following important questions on the stability of HagenPoiseuille flow in a flexible tube: (i) Is it possible to predict the numerically observed high Reynolds number unstable modes by an asymptotic analysis at high $R e$ ? (ii) Both the 'intermediate Re modes' [5] and the numerically observed unstable wall modes [6] exhibit the scaling behaviour close to $R e \sim \Sigma^{3 / 4}$ and both these modes also show the existence of a boundary layer of thickness $R e^{-1 / 3}$ where viscous effects are confined: are these two modes qualitatively similar in the limit of high $R e$ ? (iii) Can the numerically observed unstable modes exist in the limit of infinite $R e$ ? This question assumes relevance because the generalisation of classical theorems of hydrodynamic stability to inviscid flow in a flexible tube [16] predicted that the Hagen-Poiseuille flow in a flexible tube is always stable in the inviscid limit to axisymmetric disturbances. In view of this theorem, is it then possible to have unstable modes in the limit of infinite $R e$, which involve viscous effects in the fluid in an important way? In other words, are there viscous unstable solutions to the complete linear stability equations governing the fluid-flexible wall problem, which do not reduce to the inviscid solutions in the high Reynolds number limit?

The present work answers the above questions by reexamining the earlier asymptotic analysis [15] in order to determine whether the stable wall modes predicted by that analysis is a consequence of the scaling approximations made in that study. It is first useful to see how the two different scalings $R e \sim \Sigma^{3 / 5}$ and $R e \sim \Sigma^{3 / 4}$ come about from simple physical considerations. In the study of $\mathrm{Ku}-$ maran [15], the normal inertial stresses in the outer layer are balanced with the elastic stresses in the wall. As argued in [15], the normal stresses in the outer layer scale as $R e^{-1 / 3} \rho V^{2}$, where $V$ is the characteristic velocity of the laminar flow. The elastic stresses in the wall are estimated to be of the order of $G$, the shear modulus of the wall material. A balance between $R e^{-1 / 3} \rho V^{2} \sim G$ implies $\left(\rho V^{2} / G\right)^{1 / 2} \sim R e^{1 / 6}$ and it can be readily verified that $\Sigma \equiv R e^{2} /\left(\rho V^{2} / G\right) \sim R e^{5 / 3}$ and hence $R e \sim \Sigma^{3 / 5}$. Thus, a balance between the normal stresses in the outer layer and the elastic stresses in the wall yields $R e \sim \Sigma^{3 / 5}$. This scaling regime immediately implies that the tangential viscous stresses in the wall layer are $O\left(R e^{-1 / 3}\right)$ small compared to the elastic stresses in the wall medium. Indeed, the growth rates of [15] were determined without balancing the viscous shear stresses and the elastic stresses at the interface. In contrast, in the present study, the scaling regime $R e \sim \Sigma^{3 / 4}$ is considered which is obtained by a balance between the tangential viscous stresses in the 
wall layer and the elastic stresses in the wall medium at the fluid-wall interface. The viscous shear stresses in the wall layer can be estimated to be of the order of $V \eta /(\delta R)$, where $\eta$ is the viscosity of the fluid and $\delta R \sim R e^{-1 / 3} R$ is the length scale for variation in the wall layer. A balance between this viscous stress and the elastic stresses in the wall implies $V \eta R e^{1 / 3} / R \sim G$, and this yields $\left(\rho V^{2} / G\right)^{1 / 2} \sim R e^{1 / 3}$ and hence $R e \sim \Sigma^{3 / 4}$. Thus the scaling $R e \sim \Sigma^{3 / 4}$ implies a balance between the viscous shear stresses in the fluid and elastic stresses in the wall medium.

An asymptotic analysis in the limit of high $R e$ is carried out in Section 2 in the parameter regime $R e \sim \Sigma^{3 / 4}$, and this analysis shows that the wall modes in a flexible tube are indeed unstable in the limit of high $R e$. The scaling assumptions made in the present study, and consequently the ensuing asymptotic analysis, are different from that of [15] and the reason why the earlier analysis predicted only stable modes is discussed in Section 2. The results from the present asymptotic analysis show that the same analysis predicts both the numerical results of intermediate Re modes [5] and wall modes [6]: the numerical results of these earlier studies are just two of the multiple solutions predicted by the present asymptotic analysis. This result shows that the 'intermediate $R e$ modes' of [5] and the numerically predicted wall modes of [6] are qualitatively the same in the limit of high $R e$ (which also implies $\Sigma \gg 1$ ). The mechanism driving this high $R e$ instability is the transfer of energy from mean flow to fluctuations due to the deformation work done by the fluid shear stresses on the flexible surface in the wall layer (for a discussion on this point, see $[5,6])$.

The rest of this paper is organised as follows. In Section 2, an asymptotic analysis is carried out in the limit of high $R e$ for the case of stability of Hagen-Poiseuille flow in a flexible tube. Details of the asymptotic analysis are provided in Appendix A. Numerical results are presented alongside, and it is shown that the asymptotic and numerical results are in good agreement. The salient conclusions of the present study are provided in Section 3 .

\section{Analysis}

The system configuration consists of a Newtonian fluid of density $\rho$ and viscosity $\eta$ flowing through a tube of radius $R$ surrounded by a viscoelastic solid with density $\rho$, viscosity $\eta_{g}$ and coefficient of elasticity $G$ in the annular region $1<r<H$ as shown in Figure 1. Here $r$ and $x$ are the radial and axial coordinates scaled by the tube radius $R$. In this section, the lengths are scaled by $R$ and velocities by $(G / \rho)^{1 / 2}$. The base flow of interest is the Hagen-Poiseuille velocity profile:

$$
\bar{v}_{x}=\Gamma U(r)=\Gamma\left(1-r^{2}\right),
$$

where $\Gamma=\left(\rho V^{2} / G\right)^{1 / 2}$ is the nondimensional maximum velocity in the fluid, and $V$ is the dimensional maximum

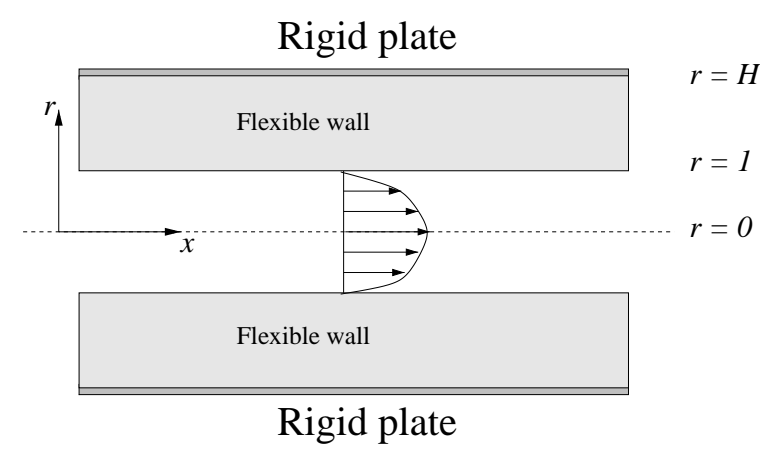

Fig. 1. Schematic diagram showing the configuration and coordinate system considered in Section 2.

velocity of the laminar flow. The non-dimensional NavierStokes equations governing the fluid motion are:

$$
\begin{aligned}
\partial_{i} v_{i} & =0, \\
\left(\partial_{t}+v_{j} \partial_{j}\right) v_{i} & =-\partial_{i} p_{f}+R e^{-1} \Gamma \partial_{j}^{2} v_{i},
\end{aligned}
$$

where the subscripts $i$ and $j$ represent components of a vector, repeated subscripts represent dot products, $\partial_{t} \equiv$ $\partial / \partial_{t}$ and $\partial_{i} \equiv \partial / \partial x_{i}$. In (2) and (3), $v_{i}$ and $p_{f}$ are the nondimensional velocity and pressure fields in the fluid respectively, and the pressure is scaled by $G$. In the above equation, $R e=R V \rho / \eta$ is the Reynolds number of the flow based on maximum fluid velocity. The stress tensor for the Newtonian fluid, scaled by $G$, is

$$
\tau_{i j}=-p \delta_{i j}+\Gamma R e^{-1}\left(\partial_{i} v_{j}+\partial_{j} v_{i}\right)
$$

The dynamics of the wall material is governed by dynamical equations for an incompressible elastic solid [17] modified to include viscous effects $[2,3,5,6,15,18]$. The dynamics of the solid wall is described by the displacement field $u_{i}$, scaled by the radius of the tube $R$, and this represents the deviation of the material points from their equilibrium positions due to the fluid stresses. In an incompressible solid, the displacement field satisfies the following solenoidal condition:

$$
\partial_{i} u_{i}=0 .
$$

The momentum balance equation for the wall material is given by:

$$
\partial_{t}^{2} u_{i}=-\partial_{i} p_{g}+\partial_{j}^{2} u_{i}+\eta_{r} \Gamma R e^{-1} \partial_{j}^{2} \partial_{t} u_{i}
$$

where $\eta_{r}=\eta_{g} / \eta$ is the ratio of the viscosities of the solid and fluid, and $p_{g}$ is the pressure in the wall material. The first term in the right-hand side is the gradient of the pressure required to satisfy incompressibility, the second term is the divergence of an elastic stress due to strain in the wall medium, while the third term is the divergence of a viscous stress. The stress tensor in the solid medium, scaled by $G$, is

$$
\sigma_{i j}=-p_{g} \delta_{i j}+\left(1+\eta_{r} \Gamma R e^{-1} \partial_{t}\right)\left(\partial_{i} u_{j}+\partial_{j} u_{i}\right) .
$$

The above form for the momentum equation and the stress tensor, incorporating frequency-independent coefficients 
of elasticity and viscosity have been used in [18] to describe the surface fluctuations on polymer gels, and in the previous stability analyses of Kumaran $[2,3,5,6,15]$ on the stability of parabolic flow in flexible tubes. The boundary conditions at the interface between the fluid and the wall material are the continuity of velocities and stresses:

$$
v_{i}=\partial_{t} u_{i}, \quad \tau_{i j}=\sigma_{i j}
$$

In the linear stability analysis, small-amplitude axisymmetric normal mode perturbations are imposed on the fluid velocity field and the displacement field in the wall:

$$
\begin{aligned}
v_{i} & =\bar{v}(r) \delta_{i x}+\tilde{v}_{i}(r) \exp [\mathrm{i} k(x-c t)], \\
u_{i} & =\tilde{u}_{i}(r) \exp [\mathrm{i} k(x-c t)] .
\end{aligned}
$$

Here $\bar{v}(r)=\Gamma U(r)=\Gamma\left(1-r^{2}\right)$ is the base flow (HagenPoiseuille) velocity profile, $x$ is the axial coordinate, $k$ a real wavenumber, $c$ is the complex wavespeed and the flow is unstable if $\operatorname{Im}[c]>0$ in the temporal stability analysis. The above form of perturbations are substituted in the governing equations in the fluid and the wall medium, and only the quantities that are linear in the perturbation variables are retained to obtain the governing linear stability equations. The resulting non-dimensional equations governing the linear stability stability of the Hagen-Poiseuille flow are given by:

$$
\begin{aligned}
\left(\mathrm{d}_{r}+r^{-1}\right) \tilde{v}_{r}+\mathrm{i} k \tilde{v}_{x}= & 0 \\
\mathrm{i} k\left(U-\frac{c}{\Gamma}\right) \tilde{v}_{x}-2 r \tilde{v}_{r}= & -\mathrm{i} k \frac{\tilde{p}_{f}}{\Gamma}+\frac{1}{R e} \\
& \times\left(\mathrm{d}_{r}^{2}+r^{-1} \mathrm{~d}_{r}-k^{2}\right) \tilde{v}_{x} \\
\mathrm{i} k\left(U-\frac{c}{\Gamma}\right) \tilde{v}_{r}= & -\frac{\mathrm{d}_{r} \tilde{p}_{f}}{\Gamma}+\frac{1}{R e} \\
& \times\left(\mathrm{d}_{r}^{2}+r^{-1} \mathrm{~d}_{r}-r^{-2}-k^{2}\right) \tilde{v}_{r} .
\end{aligned}
$$

Here, and in what follows, $\mathrm{d}_{r} \equiv \mathrm{d} / \mathrm{d} r, U(r)=\left(1-r^{2}\right)$ is the Hagen-Poiseuille velocity profile and $R e=R V \rho / \eta$ is the Reynolds number of the flow. For simplicity, the density of the wall medium and the fluid are set equal in the present work. The non-dimensional equations governing the displacement field in the wall material are given by:

$$
\begin{gathered}
\left(\mathrm{d}_{r}+r^{-1}\right) \tilde{u}_{r}+\mathrm{i} k \tilde{u}_{x}=0, \\
-\mathrm{i} k \tilde{p}_{g}+\left(1-\mathrm{i} k c \eta_{r} \Gamma R e^{-1}\right) \\
\times\left(\mathrm{d}_{r}^{2}+r^{-1} \mathrm{~d}_{r}-k^{2}\right) \tilde{u}_{x}=-k^{2} c^{2} \tilde{u}_{x}, \\
-\mathrm{d}_{r} \tilde{p}_{g}+\left(1-\mathrm{i} k c \eta_{r} \Gamma R e^{-1}\right) \\
\times\left(\mathrm{d}_{r}^{2}+r^{-1} \mathrm{~d}_{r}-r^{-2}-k^{2}\right) \tilde{u}_{r}=-k^{2} c^{2} \tilde{u}_{r} .
\end{gathered}
$$

Here $\eta_{r}=\eta_{g} / \eta$ is the ratio of viscosities of wall material and the fluid. At the interface between the fluid and the wall, it is necessary to apply the continuity of velocities and stresses. In the linear analysis, the velocity and stress fields due to the mean flow and perturbations at the perturbed interface are expanded in a Taylor series about their values at the unperturbed interface at $r=1$.

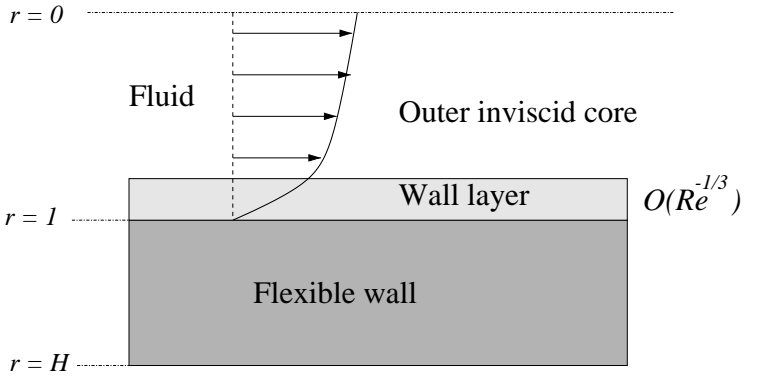

Fig. 2. Schematic diagram showing the flow structure for high Reynolds number wall modes in fluid flow through flexible tubes.

The linear terms in the series expansion are retained, and the higher order terms are neglected. This results in the following matching conditions at the interface $(r=1)$ :

$$
\begin{aligned}
\tilde{v}_{r} & =-\mathrm{i} k c \tilde{u}_{r} \\
\tilde{v}_{x}-2 \Gamma \tilde{u}_{r} & =-\mathrm{i} k c \tilde{u}_{x} \\
R e^{-1} \Gamma\left(\mathrm{d}_{r} \tilde{v}_{x}+\mathrm{i} k \tilde{v}_{r}\right)= & \left(1-\mathrm{i} k c \eta_{r} \Gamma R e^{-1}\right) \\
& \times\left(\mathrm{d}_{r} \tilde{u}_{x}+\mathrm{i} k \tilde{u}_{r}\right) \\
-\tilde{p}_{f}+2 \Gamma R e^{-1} \mathrm{~d}_{r} \tilde{v}_{r}= & -\tilde{p}_{g}+2\left(1-\mathrm{i} k c \eta_{r} \Gamma R e^{-1}\right) \mathrm{d}_{r} \tilde{u}_{r} .
\end{aligned}
$$

Here (16) and (17) are respectively the normal and tangential velocity continuity conditions at the interface. Equations (18) and (19) are respectively the tangential and normal stress continuity conditions at the interface.

In the present analysis, the limit $R e \gg 1$ is considered, and in this limit the flow in the tube can be divided into two separate regions: an 'outer layer' where viscous stresses are small compared to the inertial stresses, and a 'wall layer' near the fluid-wall interface where inertial and viscous stresses balance each other. A schematic diagram of the flow structure is shown in Figure 2. Therefore, the velocity field in the fluid is divided as

$$
\tilde{v}_{i}=\tilde{v}_{o i}+\tilde{v}_{w i} .
$$

The flow in the outer layer is obtained by setting the viscous terms in the governing equations of the fluid to zero. However, as is well known, the viscous terms in the governing equations contain the highest derivatives, and the neglect of these terms converts the momentum equations from second order to first order differential equations. Consequently, it is not possible to satisfy all the boundary conditions required for the original viscous second order differential equations, and only the normal velocity and stress conditions can be satisfied at the interface. To satisfy the tangential velocity and stress conditions, it is necessary to postulate a viscous 'wall layer' of thickness $\delta$ where viscous effects are important.

The present paper addresses a class of modes called the 'wall modes' and the wavespeed of wall modes are $O\left(R e^{-1 / 3}\right)$ small compared to the characteristic velocity of the base flow [13-15]. According to the nondimensionalisation used in the present work, this condition reduces to $c / \Gamma \sim R e^{-1 / 3}$, since $\Gamma$ is the non-dimensional 
maximum velocity of the base velocity profile. Previous numerical studies in fluid flow through flexible tubes and channels [4-6] have shown that the Reynolds number of unstable modes in the high $R e$ limit scales as $R e \sim \Sigma^{3 / 4}$, where $\Sigma=\rho G R^{2} / \eta^{2}$ is a flow-independent quantity representing the non-dimensional elasticity of the flexible wall. As discussed in the previous section, this scaling also implies that the tangential viscous stresses in the wall layer are of the same order as elastic stresses in the wall at the interface. Since $\Gamma \equiv\left(\rho V^{2} / G\right)^{1 / 2}$, it is easily seen that $\Sigma \equiv(\operatorname{Re} / \Gamma)^{2}$ and thus $\operatorname{Re} \sim \Sigma^{3 / 4}$ implies $\Gamma \sim R e^{1 / 3}$. It is therefore convenient to write $\Gamma \equiv \Gamma_{0} R e^{1 / 3}$ where $\Gamma_{0}$ is an $O(1)$ quantity. Since $c \sim R e^{-1 / 3} \Gamma$, and $\Gamma \sim R e^{1 / 3}$, it is readily seen that $c \sim 1$. Therefore, $c$ is expanded in an asymptotic series as follows:

$$
c=c^{(0)}+R e^{-1 / 3} c^{(1)}+\cdots
$$

In the limit of high $R e$, the viscous stresses are confined to a very thin layer of thickness $\delta(\ll 1)$ near the wall. The relation between the thickness of the wall layer $\delta$ and $R e$ is determined by a scaling analysis of the $x$-momentum equation of the fluid (11). To this end, it is useful to introduce an 'inner' coordinate $\xi$ such that $(1-r)=\xi \delta$. The base flow velocity profile $U(r)=(1+r)(1-r)$ is then expressed in terms of the new variable $\xi$ :

$$
U(r)=\delta \xi(2+\xi \delta) .
$$

The radial derivative $\mathrm{d}_{r}$ in the wall layer is expressed in terms of derivatives in $\xi$ as $\mathrm{d}_{r} \equiv-\delta^{-1} \mathrm{~d}_{\xi}$ (where $\left.\mathrm{d}_{\xi}=\mathrm{d} / \mathrm{d} \xi\right)$. The continuity equation (10) then indicates that $\tilde{v}_{w r}=O(\delta) \tilde{v}_{w x}$ in the wall layer. Consequently, it is convenient to expand the fluid velocities in the wall layer in the following asymptotic series:

$$
\begin{aligned}
& \tilde{v}_{w r}=\delta\left(\tilde{v}_{w r}^{(0)}+\delta \tilde{v}_{w r}^{(1)}+\cdots\right), \\
& \tilde{v}_{w x}=\tilde{v}_{w x}^{(0)}+\delta \tilde{v}_{w x}^{(1)}+\cdots
\end{aligned}
$$

The scaled continuity equation in the wall layer, to leading order in $\delta$, is then given by:

$$
-\mathrm{d}_{\xi} \tilde{v}_{w r}^{(0)}+\mathrm{i} k \tilde{v}_{w x}^{(0)}=0 .
$$

The $x$-momentum equation (11) then transforms in the wall layer as follows:

$$
\begin{array}{r}
\mathrm{i} k\left[\delta \xi(2+\xi \delta)-R e^{-1 / 3} \frac{c^{(0)}}{\Gamma_{0}}\right] \tilde{v}_{w x}^{(0)}-2 \delta(1-\xi \delta) \tilde{v}_{w r}^{(0)}= \\
-\mathrm{i} k \frac{\tilde{p}_{w f}}{R e^{1 / 3} \Gamma_{0}}+\left[\frac{\delta^{-2}}{R e} \mathrm{~d}_{\xi}^{2} \tilde{v}_{w x}^{(0)}\right] .
\end{array}
$$

In the above equation, in order to achieve a balance between the inertial term (the term in the square brackets on the left side) and the viscous term (the term in the square brackets on the right side), the small parameter $\delta$ should scale as $\delta \sim R e^{-1 / 3}$. The scaled governing equations in the wall layer and the outer layer, and the solutions for the fluid velocity field in both the layers are provided in
Appendix A of this paper. This appendix also contains the solutions to the displacement field in the wall medium.

The scaling of the boundary conditions at the interface (16-19) is considered next. The normal velocity continuity condition (16) takes the following form:

$$
\delta\left(\tilde{v}_{o r}^{(0)}+\tilde{v}_{w r}^{(0)}\right)=-\mathrm{i} k c \tilde{u}_{r} .
$$

Since $c \sim O(1)$ and the left side in the above equation is $O(\delta)$, the above equation indicates that $\tilde{u}_{r}$ at $r=1$ is $O(\delta)$. The tangential velocity condition (17) yields:

$$
\tilde{v}_{w x}^{(0)}-2 \delta^{-1} \Gamma_{0} \tilde{u}_{r}=-\mathrm{i} k c \tilde{u}_{x} .
$$

The left side of the above equation is $O(1)$ for the following reason: $\tilde{v}_{w x}$ is $O(1)$ and $\delta^{-1} \tilde{u}_{r}$ is $O(1)$ since $\tilde{u}_{r}$ at $r=1$ is $O(\delta)$. There are two ways to scale the displacement field in the wall medium. As mentioned before, $\tilde{u}_{r}$ at $r=1$ is $O(\delta)$. So, one possibility is to assume $\tilde{u}_{r} \sim O(\delta)$ through out the domain of the wall material. Then the tangential displacement in the wall $\tilde{u}_{x} \sim O(\delta)$ since in the bulk of the wall medium $\tilde{u}_{x} \sim \tilde{u}_{r}$ according to the continuity equation in the wall medium (13). When $\tilde{u}_{x} \sim O(\delta)$ in the wall material, the tangential velocity condition (28) becomes, to leading order:

$$
\tilde{v}_{w x}^{(0)}-2 \Gamma_{0} \tilde{u}_{r}^{(0)}=0 .
$$

It is shown in Appendix $B$ of this paper that the above choice of scalings for the wall displacement field yields stable wall modes identical to the ones predicted in [15] in the limit $\Lambda \ll 1$. The physical reason for the stable modes observed for this choice of scaling is discussed at the end of this section.

Another possibility to scale the displacement field in the wall is to assume $\tilde{u}_{x} \sim O(1)$ in (28) so that the tangential displacement in the wall medium enters the tangential velocity boundary condition at $r=1$. If $\tilde{u}_{x} \sim O(1)$, then $\tilde{u}_{r} \sim O(1)$ in the bulk of the wall medium since according to the continuity equation in the wall, $\tilde{u}_{x} \sim \tilde{u}_{r}$. So, the displacement field in the wall medium are expanded in an asymptotic series as:

$$
\begin{gathered}
\tilde{u}_{x}=\tilde{u}_{x}^{(0)}+\delta \tilde{u}_{x}^{(1)}+\cdots, \\
\tilde{u}_{r}=\tilde{u}_{r}^{(0)}+\delta \tilde{u}_{r}^{(1)}+\cdots
\end{gathered}
$$

The above expansions are substituted in the boundary conditions. The normal velocity boundary condition $\left(\tilde{v}_{r}=\right.$ $-\mathrm{i} k c \tilde{u}_{r}$ ) becomes:

$$
\begin{aligned}
\delta\left(\tilde{v}_{o r}^{(0)}+\tilde{v}_{w r}^{(0)}\right)= & -\mathrm{i} k\left(c^{(0)}+\delta c^{(1)}+\cdots\right) \\
& \times\left(\tilde{u}_{r}^{(0)}+\delta \tilde{u}_{r}^{(1)}+\cdots\right) .
\end{aligned}
$$

To leading order in $\delta$, the above boundary condition yields

$$
\tilde{u}_{r}^{(0)}=0 .
$$

The first correction to the normal velocity boundary condition yields

$$
\left(\tilde{v}_{o r}^{(0)}+\tilde{v}_{w r}^{(0)}\right)=-\mathrm{i} k\left(c^{(0)} \tilde{u}_{r}^{(1)}+c^{(1)} \tilde{u}_{r}^{(0)}\right) .
$$


Since $\tilde{u}_{r}^{(0)}=0$ at $r=1(32)$, the above equation becomes:

$$
\left(\tilde{v}_{o r}^{(0)}+\tilde{v}_{w r}^{(0)}\right)=-\mathrm{i} k\left(c^{(0)} \tilde{u}_{r}^{(1)}\right) .
$$

The tangential velocity boundary condition $\left(\tilde{v}_{x}-2 \Gamma \tilde{u}_{r}=\right.$ $\left.-\mathrm{i} k c \tilde{u}_{x}\right)$ becomes:

$$
\begin{aligned}
& \tilde{v}_{w x}^{(0)}-2 \delta^{-1} \Gamma_{0}\left(\tilde{u}_{r}^{(0)}+\delta \tilde{u}_{r}^{(1)}+\cdots\right)= \\
& -\mathrm{i} k\left(c^{(0)}+\delta c^{(1)}+\cdots\right)\left(\tilde{u}_{x}^{(0)}+\tilde{u}_{x}^{(1)}+\cdots\right) .
\end{aligned}
$$

To leading order in $\delta$, the above equation yields

$$
\tilde{u}_{r}^{(0)}=0 \text {. }
$$

which is identical to what was obtained (32) to leading order from the normal velocity boundary condition. The first correction to (35) is given by

$$
\tilde{v}_{w x}^{(0)}-2 \Gamma_{0} \tilde{u}_{r}^{(1)}=-\mathrm{i} k c^{(0)} \tilde{u}_{x}^{(0)} .
$$

It should be noted here that the tangential velocity in the wall layer appears only in the first correction to the tangential velocity continuity. The unscaled tangential stress condition at the interface is given by

$$
\begin{aligned}
R e^{-1} \Gamma\left[\mathrm{d}_{r} \tilde{v}_{x}+\mathrm{i} k \tilde{v}_{r}\right] & = \\
& \left(1-\mathrm{i} k c \eta_{r} \Gamma R e^{-1}\right)\left[\mathrm{d}_{r} \tilde{u}_{x}+\mathrm{i} k \tilde{u}_{r}\right] .
\end{aligned}
$$

On using the scalings for various quantities in the above equation, we obtain:

$$
\begin{aligned}
\Gamma_{0} \delta\left(-\mathrm{d}_{\xi} \tilde{v}_{w x}^{(0)}+\delta^{2} \mathrm{i} k \tilde{v}_{w r}^{(0)}\right)=\left(1-\mathrm{i} k c \eta_{r} \Gamma_{0} \delta^{2}\right) \\
\\
\times\left[\left(\mathrm{d}_{r} \tilde{u}_{x}^{(0)}+\mathrm{i} k \tilde{u}_{r}^{(0)}\right)+\delta\left(\mathrm{d}_{r} \tilde{u}_{x}^{(1)}+\mathrm{i} k \tilde{u}_{r}^{(1)}\right)\right] .
\end{aligned}
$$

To leading order, the above equation yields:

$$
\left(\mathrm{d}_{r} \tilde{u}_{x}^{(0)}+\mathrm{i} k \tilde{u}_{r}^{(0)}\right)=0 .
$$

The first correction to (39) is obtained as:

$$
\Gamma_{0}\left[-\mathrm{d}_{\xi} \tilde{v}_{w x}^{(0)}\right]=\left(\mathrm{d}_{r} \tilde{u}_{x}^{(1)}+\mathrm{i} k \tilde{u}_{r}^{(1)}\right) .
$$

The above equations show that the tangential stresses in the wall layer appears only in the first correction. The unscaled normal stress condition at the interface is given by

$$
-\tilde{p}_{f}+2 R e^{-1} \Gamma \mathrm{d}_{r} \tilde{v}_{r}=-\tilde{p}_{g}+2\left(1-\mathrm{i} k c \eta_{r} \Gamma R e^{-1}\right) \mathrm{d}_{r} \tilde{u}_{r} .
$$

As discussed in Appendix A, $\tilde{p}_{f} \sim O(1)$ to leading order. The pressure in the wall medium $\tilde{p}_{g}$ is estimated from the $x$-momentum equation in the wall (14), and this reveals that $\tilde{p}_{g}$ is $O(1)$. On using the scalings for the various quantities, the above boundary condition becomes:

$$
\begin{array}{r}
-\left(\tilde{p}_{f}^{(0)}+\delta \tilde{p}_{f}^{(1)}\right)+2 \Gamma_{0} \delta^{2}\left(-\mathrm{d}_{\xi} \tilde{v}_{w r}^{(0)}\right)=-\left(\tilde{p}_{g}^{(0)}+\delta \tilde{p}_{g}^{(1)}\right) \\
+2\left(1-\mathrm{i} k c \eta_{r} \Gamma_{0} \delta^{2}\right)\left(\mathrm{d}_{r} \tilde{u}_{r}^{(0)}+\delta \mathrm{d}_{r} \tilde{u}_{r}^{(1)}\right) .
\end{array}
$$

The leading order and the first correction equations of the above boundary condition are respectively given by

$$
\begin{aligned}
& -\tilde{p}_{f}^{(0)}=-\tilde{p}_{g}^{(0)}+2 \mathrm{~d}_{r} \tilde{u}_{r}^{(0)}, \\
& -\tilde{p}_{f}^{(1)}=-\tilde{p}_{g}^{(1)}+2 \mathrm{~d}_{r} \tilde{u}_{r}^{(1)} .
\end{aligned}
$$

The boundary conditions at $r=1$ are given below to leading order in $\delta$. As shown above, both the normal velocity (32) and tangential velocity continuity (36) at $r=1$, to leading order, reduce to:

$$
\tilde{u}_{r}^{(0)}=0 \text {. }
$$

The leading order tangential and normal stress conditions at the interface reduce, respectively, to:

$$
\begin{aligned}
\left(\mathrm{d}_{r} \tilde{u}_{x}^{(0)}+\mathrm{i} k \tilde{u}_{r}^{(0)}\right) & =0, \\
-\tilde{p}_{g}^{(0)}+2 \mathrm{~d}_{r} \tilde{u}_{r}^{(0)} & =-\tilde{p}_{o f} .
\end{aligned}
$$

At $r=H$, the displacement field should satisfy the zero displacement boundary conditions:

$$
\tilde{u}_{r}^{(0)}=0, \tilde{u}_{x}^{(0)}=0 .
$$

Note here that the velocity field in the wall layer in the fluid does not appear to leading order in the boundary conditions. This is very different from the asymptotic analysis of [15] where the wall layer quantities appeared at the leading order. To determine the leading order wavespeed, we substitute all the eigenfunctions determined previously in the above boundary conditions. This system of equations is written in a matrix form as $\mathbf{M C}=0$, where $\mathbf{C}$ is the vector of constants: $\left\{B_{1}, B_{2}, B_{3}, B_{4}, A_{1}\right\}$, and $A_{1}$ is the constant occurring in the eigenfunction of the outer layer (69) in the fluid, and $B_{1} \cdots B_{4}$ are the constants occurring in (79). The characteristic equation is obtained by setting $\operatorname{Det}[\mathbf{M}]=0$. It is easily verified that the boundary conditions $(46,47)$ and $(49)$ are independent of the constant $A_{1}$, and hence the homogeneous set of equations $(46,47)$ and $(49)$ are solved to obtain the leading order wavespeed $c^{(0)}$. Once $c^{(0)}$ is determined from this set of equations, then (48) is used to determine the amplitude of the outer velocity field in the fluid $A_{1}$. The results of this calculation indicate that there are multiple solutions for the leading order wavespeed $c^{(0)}$, all of which are positive and real, indicating that the perturbations are neutrally stable in the leading approximation.

Figure 3 illustrates the presence of multiple modes, all of which are neutrally stable in the leading order. In this figure, the $c^{(0)}$ obtained from the asymptotic analysis is plotted as straight lines, and different straight lines denote the different neutrally stable modes obtained in the leading order. The asymptotic results for $c^{(0)}$ are compared with the wavespeed $c_{\text {num }}$ obtained from the neutral stability results of a full numerical solution of the governing equations at different values of $R e$ ranging from $10^{3}$ to $10^{6}$. The numerical method employed to obtain these results is explained a little later. Figure 3 shows that the asymptotic and numerical results are in good agreement, and 


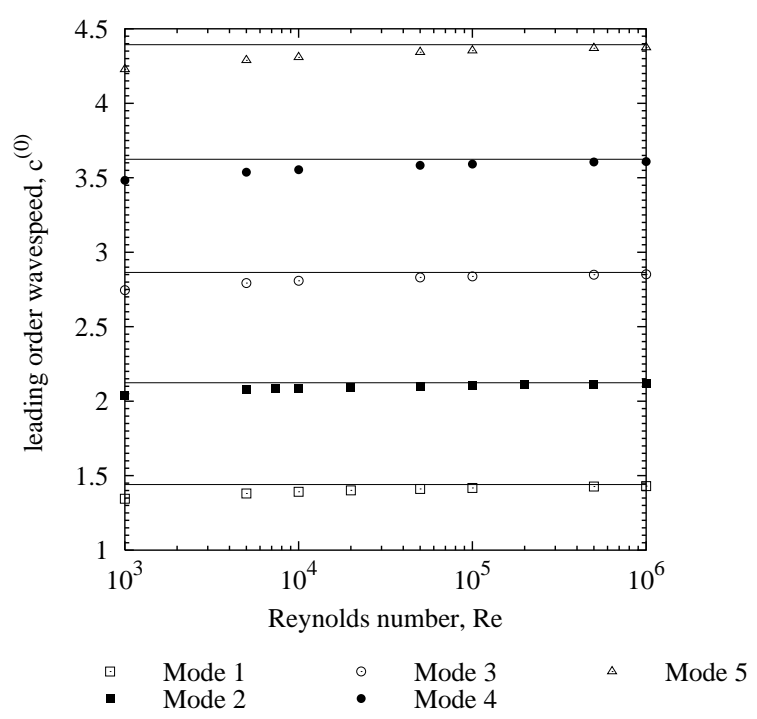

Fig. 3. Comparison of the asymptotic results (lines) obtained from the present analysis with the numerical results (symbols): Variation of the leading order wavespeed $c^{(0)}$ with the Reynolds number $R e$ for the different multiple solutions: $H=5, k=$ $1, \eta_{r}=0$.

the numerical results also show the existence of multiple harmonics in the wavespeed. Moreover, the high Reynolds number asymptotic wavespeed $c^{(0)}$ is well predicted by the numerical method even when $R e=10^{3}$, and the small parameter $\delta=R e^{-1 / 3}=0.1$ for $R e=10^{3}$. Figure 4 shows the error in the asymptotic wavespeed $c^{(0)}$ when compared with the wavespeed obtained from the full numerical solution. The asymptotic analysis carried out above predicts that the first correction to the wavespeed is $O\left(R e^{-1 / 3}\right)$ smaller than the leading wavespeed $c^{(0)}$, and hence $c^{(0)}$ is in error by $O\left(R e^{-1 / 3}\right)$. This prediction of the asymptotic analysis is in good agreement with the numerical results presented in Figure 4, which shows that the relative error (defined as $\left|c_{\text {num }}-c^{(0)}\right| / c_{\text {num }}$ ) between asymptotic $\left(c^{(0)}\right)$ and numerically obtained $\left(c_{\text {num }}\right)$ wavespeeds indeed decreases as $R e^{-1 / 3}$.

Since the flow is neutrally stable in the leading approximation, it is necessary to calculate the next correction to the wavespeed $c^{(1)}$ in order to determine the stability of the system. The first correction $c^{(1)}$ is calculated from the $O(\delta)$ correction to the characteristic equation $\operatorname{Det}(\mathbf{M})=0$. This correction to $\operatorname{Det}(\mathbf{M})$ is determined by substituting the displacement eigenfunctions correct to $O(\delta)$ (i.e. both the leading order and first correction to $\left.\tilde{u}_{r}, \tilde{u}_{x}\right)$ in the boundary conditions. It is also necessary to determine the first corrections to the outer layer fluid pressure $\tilde{p}_{o f}^{(1)}$. However, the leading order contributions to the wall layer quantities (57) are sufficient for this level of approximation. All the eigenfunctions are then inserted into the boundary conditions, and the determinant of the characteristic matrix $\operatorname{Det}(\mathbf{M})$ is expanded in $\delta$. It should be noted that the tangential velocity continuity condition at the interface and the fluid tangential stresses in the wall layer appear only at the $O(\delta)$ correction to the characteristic equation. The ratio of wall to

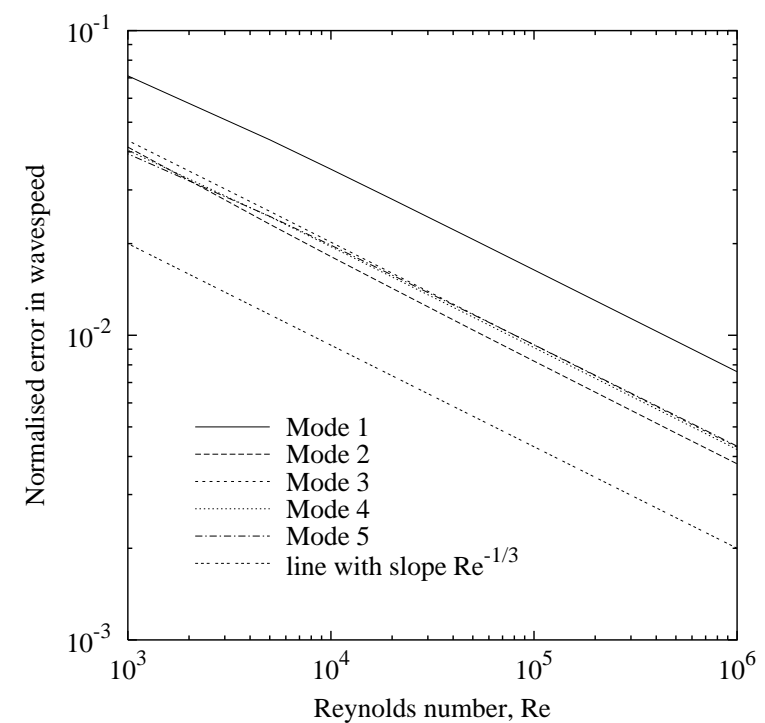

Fig. 4. Variation of error in the leading order wave speed $c^{(0)}$ determined from the asymptotic analysis with Reynolds number $R e$ for the different multiple solutions: $H=5, k=$ $1, \eta_{r}=0$.

fluid viscosities $\eta_{r}$ is considered to be an $O(1)$ quantity, and hence does not appear at the first correction to the characteristic equation. The leading order determinant of this expansion yields an expression for $c^{(0)}$ which was already determined, and the first correction to the determinant in the small parameter $\delta$ yields an expression for $c^{(1)}$. The expression for $c^{(1)}$ is solved from the first correction to the characteristic equation, and it turns out that $c^{(1)}$ is a complex quantity. The flow is unstable if $\operatorname{Im}\left[c^{(1)}\right]>0$ and stable if $\operatorname{Im}\left[c^{(1)}\right]<0$, and so $\operatorname{Im}\left[c^{(1)}\right]$ is set to zero to determine the scaled velocity $\Gamma_{0}$ required for neutrally stable modes. As mentioned before, the leading order characteristic equation admits multiple real solutions for $c^{(0)}$. For each of the multiple solutions $c^{(0)}$ there is a first correction $c^{(1)}$, and each of these $c^{(1)}$ is solved to determine neutrally stable modes. Hence, corresponding to the multiple solutions to $c^{(0)}$, there are multiple solutions for the scaled velocity $\Gamma_{0}$ required for neutrally stable modes, and if $\Gamma>\Gamma_{0} R e^{1 / 3}$ the flow is unstable. Thus the first correction to the wavespeed shows that there are multiple unstable solutions in the limit of high Re. Figure 5 shows the comparison between asymptotic and numerical results for $\Gamma_{0}$, for the different multiple solutions obtained from the asymptotic analysis. It should be recalled here that $\Gamma_{0}=\Gamma R e^{-1 / 3}$ is the scaled maximum velocity of the base flow. In Figure 5 , if $\Gamma>\Gamma_{0} R e^{1 / 3}$ then the flow is unstable. This figure again shows that there is good agreement between asymptotic and numerical results for $\Gamma_{0}$ even at $R e=10^{3}$. Figure 6 shows that the relative difference between the asymptotic and numerical results for $\Gamma_{0}$ decreases as $R e^{-1 / 3}$, as predicted by the asymptotic analysis.

The numerical scheme used to solve the complete differential equations and boundary conditions governing the stability of the system (Eqs. (10-19)) is briefly outlined here. The method is identical to that used in our 


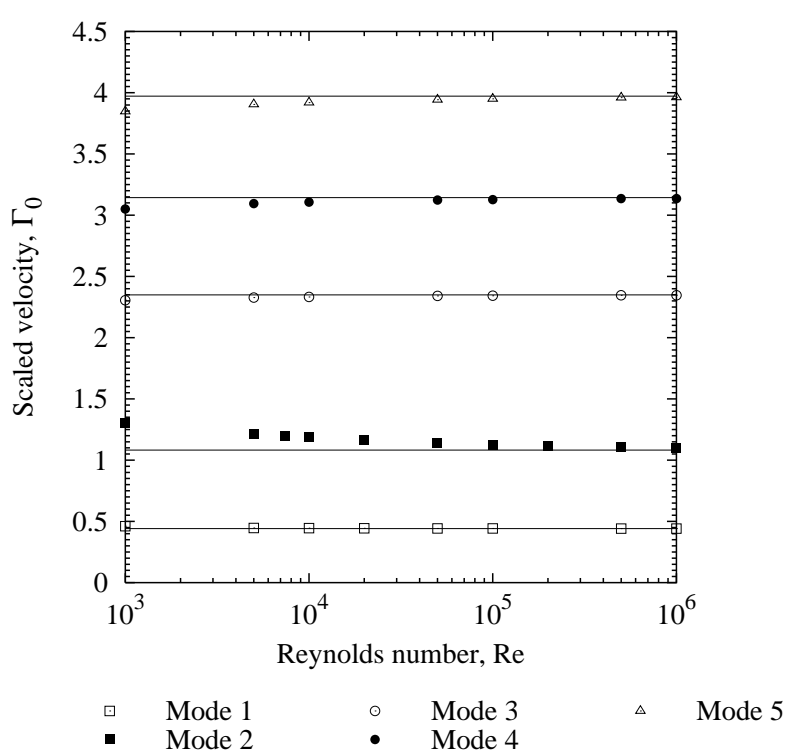

Fig. 5. Comparison of the asymptotic results (lines) obtained from the present analysis with the numerical results (symbols): Variation of the scaled fluid velocity $\Gamma_{0} \equiv \Gamma R e^{-1 / 3}$ with the Reynolds number $R e$ for the different multiple solutions: $H=$ $5, k=1, \eta_{r}=0$.

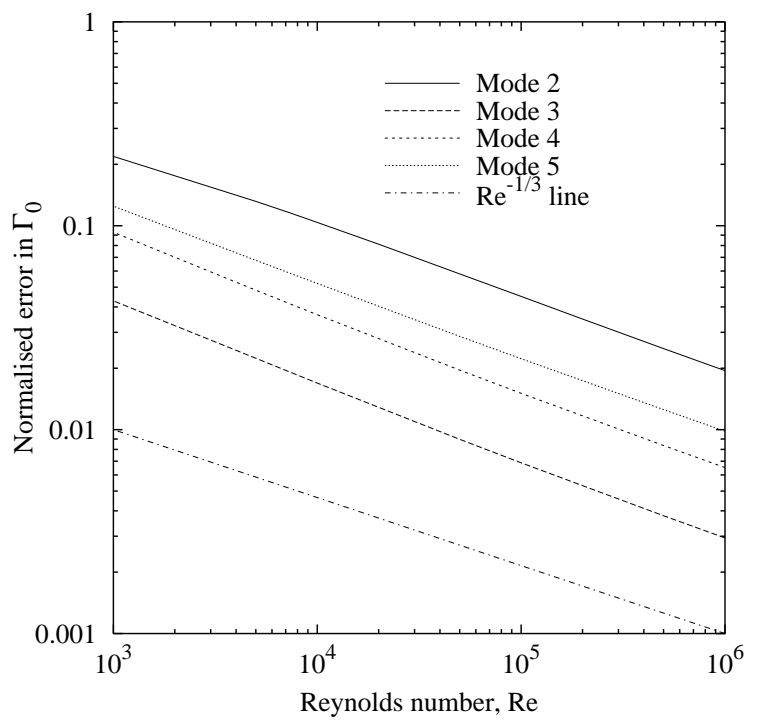

Fig. 6. Variation of error in the scaled velocity $\Gamma_{0}$ determined from the asymptotic analysis with Reynolds number $R e$ for the different multiple solutions: $H=5, k=1, \eta_{r}=0$.

previous studies on fluid flow through flexible tubes $[5,6$, 19]. The governing equations for the velocity field in the fluid and the displacement field in the flexible wall are reduced to two fourth-order differential equations. There are two linearly independent solutions for the velocity field in the fluid which are consistent with the symmetry conditions at the centre of the tube. For $r \rightarrow 0$, the linearly independent solutions are obtained as a Frobenius series in $r$. This series solution is extended up to a small but finite value of $r$. Using these as the initial conditions, the governing stability equations are numerically integrated to the fluid-wall interface at $r=1$ using a fourth order Runge-Kutta scheme with adaptive step size control. A

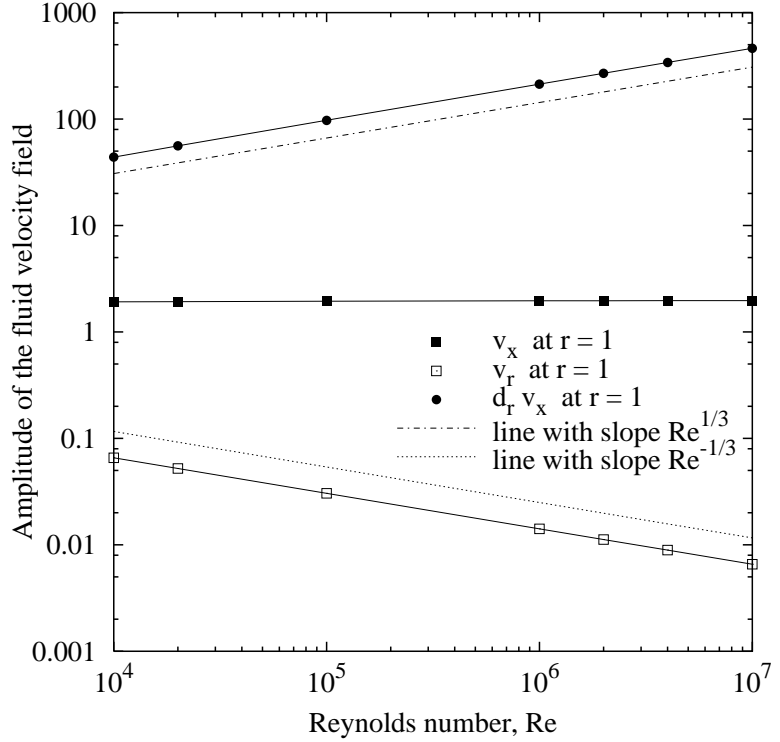

Fig. 7. Variation of the absolute value of velocity field in the fluid with Reynolds number $R e$ for the continuation of viscous mode [5] : Data from full numerical solution for $H=2, k=$ $1, \eta_{r}=0$.

Gram-Schmidt orthonormalisation procedure was implemented in order to overcome the stiff nature of the governing equations in the limit of high $R e$. There are two linearly independent solutions for the displacement field in the wall consistent with the zero displacement conditions at $r=H$. Both of these are determined using a fourthorder Runge-Kutta technique. The solutions for the fluid velocity and wall displacement fields at the interface are inserted into the boundary conditions to obtain the characteristic matrix. The characteristic equation is obtained by setting the determinant of the matrix to zero, and this is solved to determine the wavespeed $c$. The characteristic equation is a highly nonlinear equation, and the high Reynolds number asymptotic results obtained in this section are used as starting guesses, and an iterative NewtonRaphson method was used to obtain the wavespeed. This numerical method was validated extensively in our previous studies $[5,6,19]$, by comparing the numerical results with the different asymptotic results that exist in the stability of flow through flexible tubes, as well as with the known numerical results in the case of stability of fluid flow in rigid tubes.

It is instructive to examine the eigenfunctions of the neutral modes obtained from the full numerical solution, in order to verify whether the scaling assumptions made in the asymptotic analysis for the fluid velocities and wall displacements are consistent with the numerical solution. The eigenfunctions are calculated using the normalisation condition that the absolute value of the $\tilde{v}_{r}$ eigenfunction at the fluid-wall interface satisfies $\left|\tilde{v}_{r}\right|_{r=1}=\sqrt{2} R e^{-1 / 3}$. Figure 7 shows the variation of various dynamical quantities in the fluid evaluated at $r=1$ with the Reynolds number. By construction, $\left|\tilde{v}_{r}\right|_{r=1}$ scales as $R e^{-1 / 3}$ (23). The figure shows that $\left|\tilde{v}_{x}\right|$ is an $O(1)$ quantity (compare with the asymptotic expansion 24$)$, and $\left|d_{r} \tilde{v}_{x}\right|$ indeed 


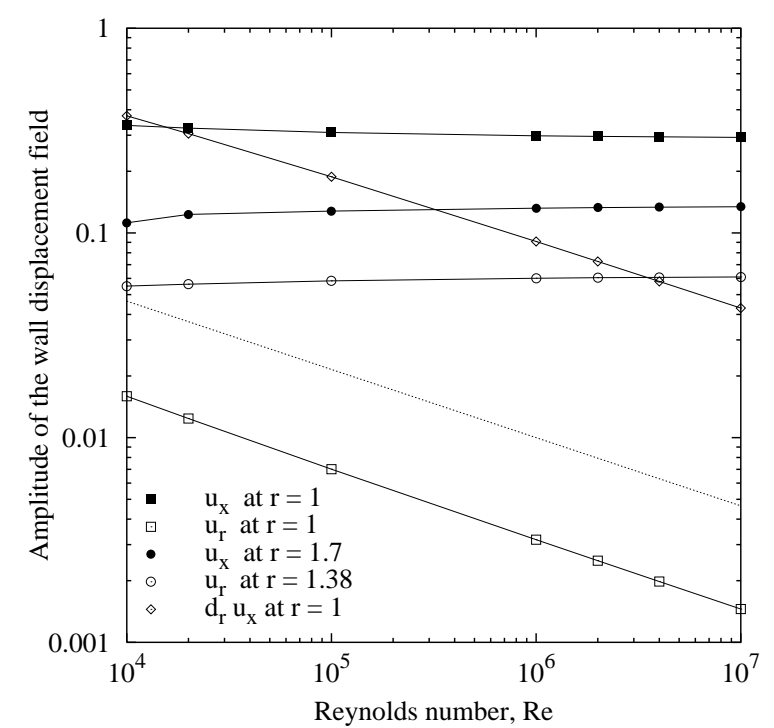

Fig. 8. Variation of the absolute value of displacement field in the wall material with Reynolds number Re for the continuation of viscous mode [5]: Data from full numerical solution for $H=2, k=1, \eta_{r}=0$. The dotted line is the reference straight line with slope $R e^{-1 / 3}$.

scales as $R e^{1 / 3}$ as was anticipated in the asymptotic analysis. Thus, the results from the full numerical solution for the eigenfunctions of various dynamical quantities in the fluid are consistent with the scaling assumptions made in the asymptotic analysis. Figure 8 shows the variation of the wall displacement field with $R e$. This figure clearly shows that $\left|\tilde{u}_{r}\right|_{r=1}$ scales as $O\left(R e^{-1 / 3}\right)$ (see Eq. (27)), while $\left|\tilde{u}_{r}\right|$ evaluated at some interior point in the wall medium is $O(1)$ (see Eq. (30)). Both these numerical results are in agreement with the scaling assumptions made in the asymptotic analysis. Moreover, as anticipated in the asymptotic analysis, $\left|\tilde{u}_{x}\right|$ is $O(1)$ both at $r=1$ and at some interior point in the wall medium. Importantly, even though $\left|\tilde{u}_{x}\right|$ is $O(1)$ at $r=1,\left|d_{r} \tilde{u}_{x}\right|$ scales as $O\left(R e^{-1 / 3}\right)$ at the interface. This result is again consistent with the outcome of the asymptotic analysis, which indicated that the tangential stresses of the wall at the interface is zero to leading order (see Eq. (40)), and the next correction to the tangential stresses at the interface is $O\left(R e^{-1 / 3}\right)$ (see Eq. (39)). Therefore, the results for eigenfunctions from the full numerical solution are consistent with the scaling assumptions of the asymptotic analysis. Figure 9 shows the variation of the thickness of the wall layer thickness $\delta$ with the Reynolds number. The wall layer thickness may be estimated by computing the ratio $\tilde{v}_{x} / d_{r} \tilde{v}_{x}$ from the full numerical solution. As Figure 9 shows, the wall layer thickness $\delta$ decreases as $R e^{-1 / 3}$ in the limit of large $R e$ as predicted by the asymptotic analysis, and this shows that the numerically observed modes are indeed the wall modes. Figures 10, and 11 show the variation of $\left|\tilde{v}_{r}\right|$ and $\left|\tilde{v}_{x}\right|$ in the fluid with $r$, and here the eigenfunctions are calculated subject to the normalisation condition that $\left|\tilde{v}_{r}\right|=\sqrt{2}$ at $r=1$. These figures show that the velocities are large very close to the wall $(r=1)$, and there is a small region near the wall where the fluid velocity varies

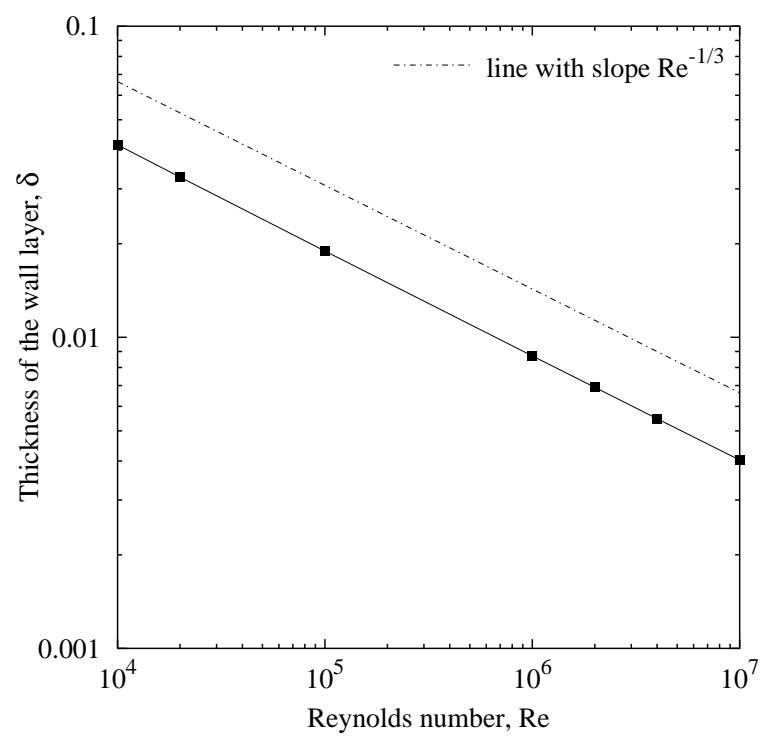

Fig. 9. Variation of the thickness of the wall layer $\delta$ with Reynolds number $R e$ for the continuation of viscous mode [5]: Data (symbols) from full numerical solution for $H=2, k=$ $1, \eta_{r}=0$.

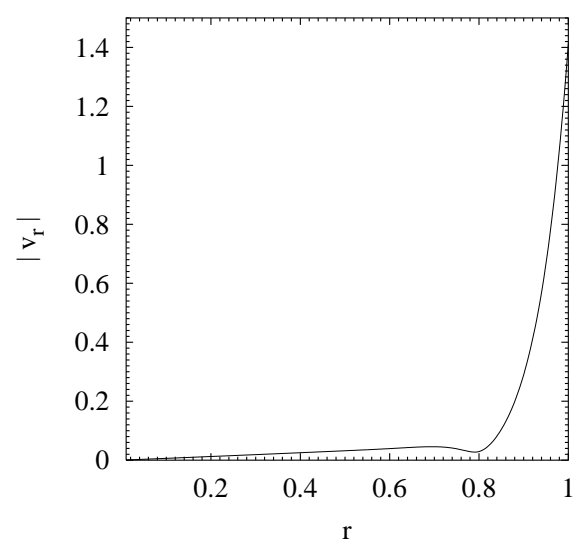

Fig. 10. The absolute value of the $\tilde{v}_{r}$ eigenfunction obtained from the full numerical calculation showing that the variation of the fluid velocity is confined to a small region near the wall: $R e=10^{3}, H=2, k=1, \eta_{r}=0$.

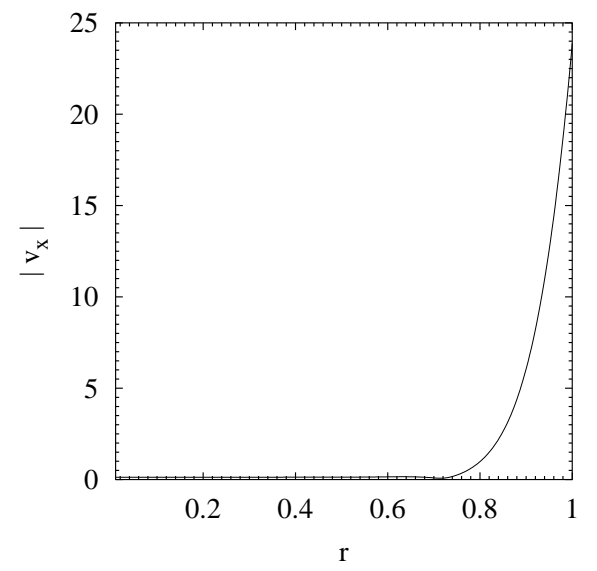

Fig. 11. The absolute value of the $\tilde{v}_{x}$ eigenfunction obtained from the full numerical calculation showing that the variation of the fluid velocity is confined to a small region near the wall: $R e=10^{3}, H=2, k=1, \eta_{r}=0$. 


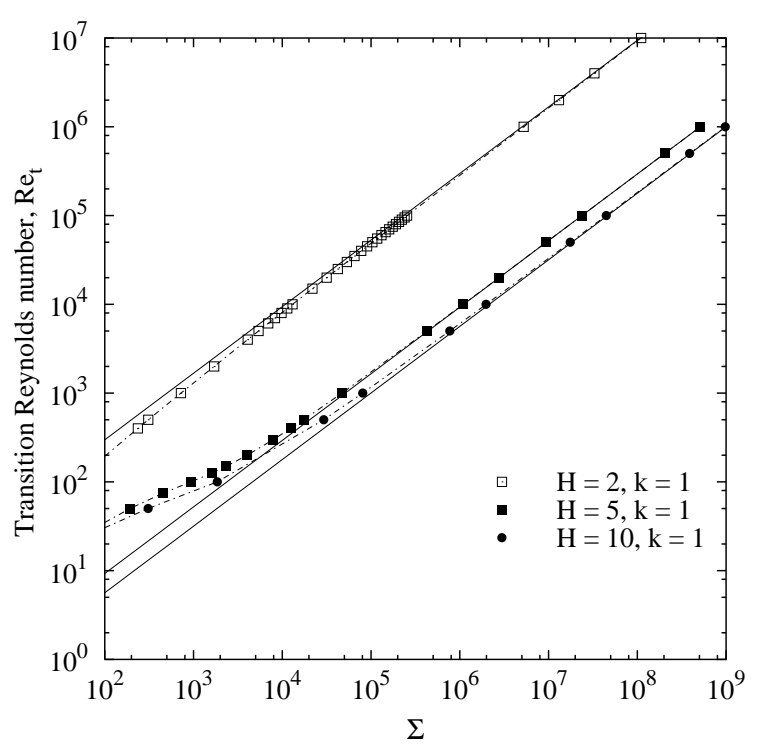

Fig. 12. Comparison of the asymptotic results (lines) obtained from the present analysis with the numerical results (dotted lines with symbols) for intermediate Reynolds number modes of [5]. $\eta_{r}=0$ for all the cases plotted.

rapidly. Both these observations indicate that the numerical eigenfunctions clearly exhibit the behaviour that is characteristic of wall modes.

The results obtained from the asymptotic analysis are now compared with the previous numerical results of [5] and [6]. As mentioned before, the asymptotic analysis yields multiple unstable modes, and each of these modes are characterised by the leading order wavespeed $c^{(0)}$ and the scaled velocity required for instability $\Gamma_{0}$. The comparison of the present asymptotic results with the previous numerical results reveals that the $c^{(0)}$ (which is a real quantity) with the lowest magnitude (called the 'first harmonic') and the $\Gamma_{0}$ necessary for instability correspond to the intermediate Reynolds number mode of [5]. It should be recalled here that [5] continued numerically the zero Reynolds number unstable mode of [2] to intermediate Reynolds number. Figure 12 shows the comparison of asymptotic results of the present study with the numerical results of [5], where the Reynolds number required for instability is plotted as a function of the non-dimensional parameter $\Sigma$. This comparison shows that the previous numerical results are accurately captured by the present asymptotic results, and the Reynolds number required for unstable modes scales as $R e \propto \Sigma^{3 / 4}$. The next higher harmonic of the asymptotic results, i.e. the $c^{(0)}$ with magnitude higher than the first harmonic, and the $\Gamma_{0}$ necessary for instability, correspond to the numerical results of [6]. It should be recalled that [6] continued numerically the stable wall mode of [15] to intermediate Reynolds number, and found that the wall mode becomes unstable in that parameter regime. Figure 13 shows the comparison between the present asymptotic results with the numerical results of [6]. This again shows that the asymptotic results are in good agreement with the previous numerical results. Though the comparison between the present asymptotic

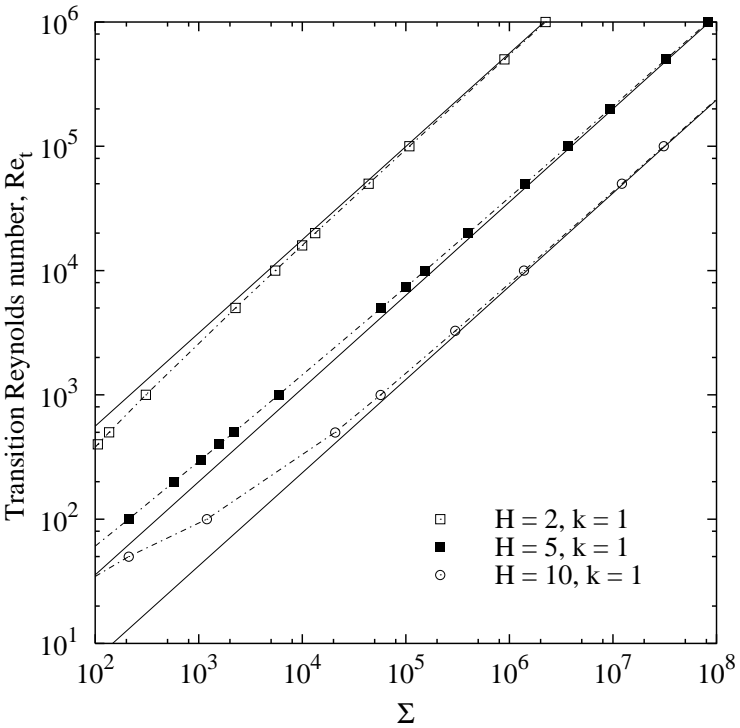

Fig. 13. Comparison of the asymptotic results (lines) obtained from the present analysis with the numerical results (dotted lines with symbols) of [6] for continuation of wall modes. $\eta_{r}=0$ for all the cases plotted.

and previous numerical results is shown only for a few parameters in these figures, it was ascertained that the asymptotic analysis accurately predicts the numerical results for all the results reported in $[5,6]$. Also, it was always found that the lowest harmonic from the present asymptotic analysis corresponds to the result of [5], and the next higher harmonic of the present analysis corresponds to the result of $[6]$.

More importantly, the results from the present asymptotic analysis captures both the numerical results of [5] and [6]. Thus the present study unifies the numerically observed unstable modes for Hagen-Poiseuille flow in a flexible tube into a single class of modes, namely the high Reynolds number wall modes. There are of course, multiple unstable modes predicted by the present asymptotic analysis as shown in Figure 5, but the lowest harmonic for $c^{(0)}$ corresponds to the mode with lowest $\Gamma_{0}$, and hence this is the most unstable mode. The high Reynolds number results of [5] were obtained as a continuation of the zero Reynolds number unstable modes, while the high Reynolds number results of $[6]$ were obtained as a numerical continuation of the stable wall modes. Since the present asymptotic analysis shows that both these previous numerical results are qualitatively similar in the limit of $R e \gg 1$, it is of interest to examine the nature of the numerical continuation of these high Reynolds number wall modes for low Reynolds numbers. Figure 14 shows the results from the numerical continuation of different neutral modes predicted by the high Reynolds number asymptotic analysis. There are, of course, more unstable modes, but only the first five harmonics are plotted here for clarity. At $R e \gg 1$, all these modes obey the scaling behaviour $R e \sim \Sigma^{3 / 4}$, and all these modes are the continuations of the present asymptotic analysis. The lowest harmonic (termed Mode 1 in the figure) continues to the limit of zero 


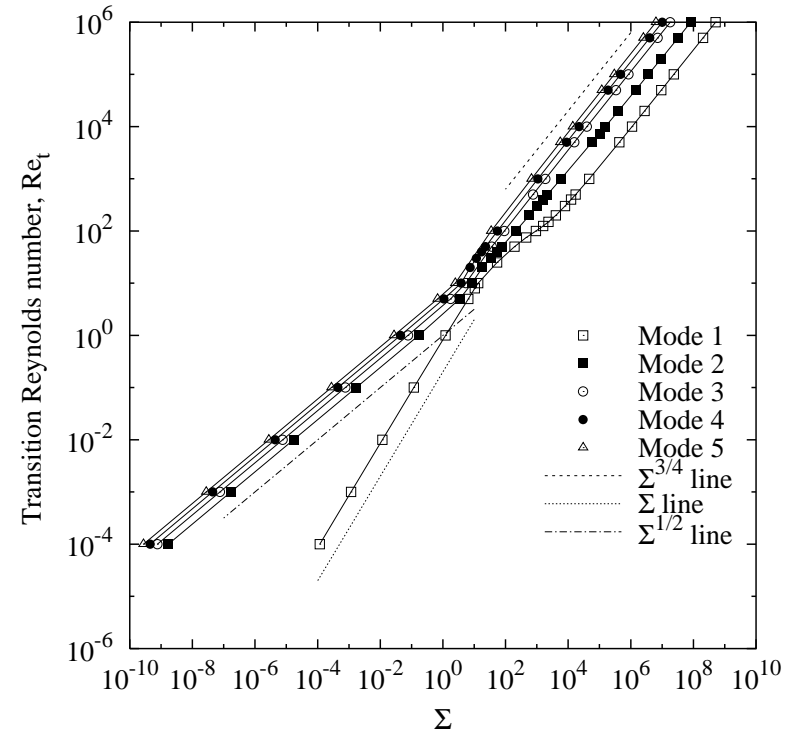

Fig. 14. The neutral stability curves for different modes obtained from the numerical continuation of the asymptotic results: $R e_{t}$ vs. $\Sigma$ for $H=5, k=1, \eta_{r}=0$. The dotted lines are reference lines showing different scaling behaviour.

Reynolds number, and the scaling behaviour at $R e \ll 1$ is given by $R e \sim \Sigma$. This mode continues to the zero Reynolds number viscous mode of [2], and this mode also corresponds to the intermediate Reynolds number results of [5] in the $R e \gg 1$ regime. The higher harmonics (termed Modes 2 to 5 ) in Figure 14 all show the scaling behaviour $R e \sim \Sigma^{3 / 4}$ for $R e \gg 1$, and this behaviour is similar to that of the lowest harmonic Mode 1 at $R e \gg 1$. However, as shown in Figure 14, for $R e \ll 1$, the higher harmonics show the scaling behaviour $R e \sim \Sigma^{1 / 2}$ which is very different from that of Mode 1 . This class of modes with $R e \sim \Sigma^{1 / 2}$ for $R e \ll 1$ are qualitatively different from the 'viscous mode' [2]. However, for a given set of fluid and wall parameters, i.e. for a fixed $\Sigma$, the viscous mode (Mode 1) becomes unstable at much lower Re than the other modes (Modes 2 to 5 and the other higher harmonics that are not shown in this figure), and hence the Mode 1 of the high- $R e$ asymptotic analysis is the most unstable mode even in the low $R e$ regime.

The physical mechanism driving the wall mode instability can be explained using an energy balance analysis, as was done in the previous numerical studies $[5,6]$. It was shown in those studies that there is an instability when the transfer of energy from mean flow to fluctuations due to the shear work done by the fluid at the flexible surface is larger than the rate of viscous dissipation of energy in the wall layer. It is also useful to note that the wall mode instability analysed in this study is not a continuation of the wall modes in a rigid tube because the transition Reynolds number for unstable wall modes in a flexible tube scales as $\left(\rho R^{2} G / \eta^{2}\right)^{3 / 4}$ in the limit of large wall elasticity, i.e. the rigid tube limit, whereas the wall modes in a rigid tube are stable in the same limit.

Before closing this section, it is useful to summarise the important qualitative differences between the present asymptotic analysis and the previous high-Re asymptotic analysis of Kumaran [15]:

1. The analysis of Kumaran [15] mainly focussed on the regime $\Lambda \sim 1$, where $\Lambda \equiv R e^{1 / 3} G /\left(\rho V^{2}\right)$, and $\Lambda \sim 1$ implies $R e \sim \Sigma^{3 / 5}$. This scaling implies that the normal stresses in the outer layer are of the same order as the elastic stresses in the wall medium, but the tangential viscous stresses are subdominant compared to the elastic stresses at the interface. In contrast, the present asymptotic analysis directly accesses the scaling regime $R e \sim \Sigma^{3 / 4}$ observed in numerical studies, which implies $\Lambda \sim R e^{-1 / 3}$, i.e. $\Lambda \ll 1$ for $R e \gg 1$. This scaling regime implies that the tangential viscous stresses are balanced by the elastic stresses of the wall at the interface. In [15], the results for the limiting case $\Lambda \ll 1$ showed that the wall modes are stable in a flexible tube, while the present analysis shows that the wall modes are unstable in the same limit $\Lambda \sim R e^{-1 / 3} \ll 1$ using a different set of scaling assumptions for the wall displacement field.

2. In the analysis of Kumaran [15], the tangential displacement term is sub-dominant in the tangential velocity condition (28) and in the present study (see Appendix B) it is shown that it is this assumption which resulted in the stable modes reported in that study. For this scaling, the wall medium is 'tangentially rigid' since the tangential displacement field in the wall is sub-dominant compared to the tangential fluid velocity in the wall layer. Since the flexible tube becomes 'tangentially rigid', there is no possibility of transfer of energy from mean flow to fluctuations through shear deformation work done by the fluid at the interface. Whereas, for the unstable wall modes analysed in the present work, the tangential displacement term present in the right-hand side of (37) is of the same order as the other two terms in the tangential velocity boundary condition, and hence there is a possibility of energy transfer from mean flow to fluctuations thereby rendering the flow unstable.

3. In the present work, both the inertial and elastic terms are of the same order in the wall medium, while in [15], the inertial stresses in the wall medium are $O\left(R e^{-1 / 3}\right)$ smaller than the elastic stresses, and hence do not enter the analysis in the leading order.

4. In the present work, the wall layer quantities and fluid shear stresses do not appear in the determination of the leading order wavespeed $c^{0}$, while they appear at the leading order in the analysis of Kumaran [15].

\section{Concluding remarks}

Previous numerical studies on the stability of HagenPoiseuille flow in a flexible tube showed that the flow could be unstable at high Reynolds number, while previous high Reynolds number asymptotic analyses (that of both wall modes and inviscid modes) predict only stable modes. This discrepancy prompted the present asymptotic analysis which re-examined the asymptotic analysis of wall 
modes with a fresh set of scaling assumptions. The present asymptotic analysis shows that the Hagen-Poiseuille flow does become unstable in the limit of high $R e$ in the parameter regime $\operatorname{Re} \propto \Sigma^{3 / 4}$, which is the regime where the previous numerical studies revealed unstable modes. The present analysis unifies many of the numerically known unstable modes at high $R e[5,6]$ into one class of modes, viz., high Reynolds number wall modes, where the viscous effects in the fluid are confined in a layer of thickness $O\left(R e^{-1 / 3}\right)$ near the wall. Thus, the present work explains why there exist unstable modes at high Reynolds number in fluid flow through flexible tubes where viscous effects in the fluid are confined in a small region of thickness $O\left(R e^{-1 / 3}\right)$ smaller than the tube radius. Moreover, the instability obtained in the present asymptotic analysis is not a continuation of the wall modes in a rigid tube, because the critical Reynolds number for the wall mode instability in a flexible tube is proportional to $\left(\rho G R^{2} / \eta^{2}\right)^{3 / 4}$ in the limit of large wall elasticity (i.e. the rigid tube limit), whereas the wall modes in a rigid tube are always stable. The physical mechanism driving the wall mode instability in flexible tubes is the transfer of energy from the mean flow to the fluctuations due to the deformation work done by the mean flow in the wall layer on the flexible surface. When this shear work done by the fluid at the surface is greater than the rate of viscous dissipation of energy in the wall layer, the flow becomes unstable.

\section{Appendix A}

In this appendix, the details of the asymptotic analysis of the fluid governing equations are provided, and the solutions for the fluid velocity field in the outer and wall layers are derived. The solutions for the leading order and first correction to the displacement field in the wall medium are also provided here.

Without loss of generality, the small parameter $\delta$ of the asymptotic analysis can be defined as $\delta=R e^{-1 / 3}$. In the $x$ momentum equation (26), in order for the pressure in the wall layer to be of the same magnitude as the other terms, we require $\tilde{p}_{w f} \sim O(1)$ and hence $\tilde{p}_{w f}$ is expanded as: $\tilde{p}_{w f}=\tilde{p}_{w f}^{(0)}+\cdots$. The scaled $x$-momentum equation in the wall layer, to leading order in the small parameter $\delta$, is given by:

$$
\mathrm{i} k\left[2 \xi-\frac{c^{(0)}}{\Gamma_{0}}\right] \tilde{v}_{w x}^{(0)}-2 \tilde{v}_{w r}^{(0)}=-\mathrm{i} k \frac{\tilde{p}_{w f}^{(0)}}{\Gamma_{0}}+\mathrm{d}_{\xi}^{2} \tilde{v}_{w x}^{(0)}
$$

The $r$-momentum equation (12) in the fluid is scaled similarly to obtain the following equation:

$$
\delta^{2} \mathrm{i} k\left[2 \xi-\frac{c^{(0)}}{\Gamma_{0}}\right] \tilde{v}_{w r}^{(0)}=\delta^{-1} \mathrm{~d}_{\xi} \tilde{p}_{w f}^{(0)}+\delta^{2} \mathrm{~d}_{\xi}^{2} \tilde{v}_{w r}^{(0)} .
$$

To leading order in $\delta$ the above equation reduces to $\mathrm{d}_{\xi} \tilde{p}_{w f}^{(0)}=0$. A single governing equation for $\tilde{v}_{w x}^{(0)}$ in the wall layer is obtained by differentiating (50) with respect to $\xi$ and on using the continuity equation (25) and the condition $\mathrm{d}_{\xi} \tilde{p}_{w f}^{(0)}=0$ :

$$
\left[\mathrm{d}_{\xi}^{2}-\mathrm{i} k\left(2 \xi-\frac{c^{(0)}}{\Gamma_{0}}\right)\right] \mathrm{d}_{\xi} \tilde{v}_{w x}^{(0)}=0 .
$$

It is convenient to define another variable

$$
y=(2 \mathrm{i} k)^{1 / 3}\left[-c^{(0)} /\left(2 \Gamma_{0}\right)+\xi\right],
$$

and the general solution of the above equation (52) in terms of this new variable $y$ is given by

$$
\tilde{v}_{w x}^{(0)}=C_{1}+C_{2} A i(y, 1)+C_{3} B i(y, 1) .
$$

Here $A i(y, 1)$ and $B i(y, 1)$ are the generalised Airy functions [20]:

$$
\begin{aligned}
& A i(y, 1)=\int_{\infty}^{y} \mathrm{~d} y \operatorname{Ai}(y), \quad A i(y,-1)=\mathrm{d}_{y} A i(y), \\
& B i(y, 1)=\int_{\infty}^{y} \mathrm{~d} y B i(y), \quad B i(y,-1)=\mathrm{d}_{y} B i(y),
\end{aligned}
$$

and $A i(y)$ and $B i(y)$ are the Airy functions which are the solutions of the Airy equation $\left(\mathrm{d}_{y}^{2}+y\right) \psi(y)=0$. The constants $C_{1}, C_{2}$ and $C_{3}$ in (54) are determined by matching the solution for the tangential velocity in the wall layer with the tangential velocity in the outer layer. It is shown below (Eq. (64)) that the tangential velocity in the outer layer is $O(\delta)$ smaller than the tangential velocity in the wall layer, and hence the matching condition requires that $\tilde{v}_{w x}^{(0)} \rightarrow 0$ for $\xi \rightarrow \infty$. The Airy functions $A i(y, p)$ are convergent in the limit $\xi \rightarrow \infty$ only for $(-\pi / 3)<\operatorname{Arg}(\xi)<(\pi / 3)$, and so it is necessary to choose $\operatorname{Arg}\left(\mathrm{i}^{1 / 3}\right)=\pi / 6$ in (53). In this domain, the Airy function $B i(y, p)$ diverges, and so the matching condition with the outer layer requires that $C_{1}=0$ and $C_{3}=0$. As a result, the solution for the velocities and pressure in the wall layer is given by

$$
\begin{aligned}
& \tilde{v}_{w x}^{(0)}=C_{2} A i(y, 1) \\
& \tilde{v}_{w r}^{(0)}=C_{2} 2^{-1 / 3}(\mathrm{i} k)^{2 / 3}[y A i(y, 1)-A i(y,-1)], \\
& \tilde{p}_{w f}^{(0)}=0 .
\end{aligned}
$$

The leading order fluid pressure in the wall layer $\tilde{p}_{w f}^{(0)}$ is identically zero, and so it is necessary to estimate the next dominant contribution to the fluid pressure in the wall layer. The next dominant contribution to the fluid pressure in the wall layer is obtained from the $r$-momentum balance (12) which shows that the next highest contribution is $O\left(\delta^{2}\right)$ smaller than $\tilde{v}_{w x}$. For future reference, the magnitudes of various dynamical quantities in the wall layer are:

$$
\tilde{v}_{w x} \sim 1, \quad \tilde{v}_{w r} \sim \delta, \quad \tilde{\tau}_{w r r} \sim \delta^{2}, \quad \tilde{\tau}_{w x r} \sim \delta .
$$

The equations for the inviscid outer flow in the tube are obtained by formally setting $R e^{-1}=0$ in the governing 
equations of the fluid (11) and (12):

$$
\begin{aligned}
\left(\mathrm{d}_{r}+r^{-1}\right) \tilde{v}_{o r}+\mathrm{i} k \tilde{v}_{o x} & =0 \\
\mathrm{i} k\left(U-\frac{c}{\Gamma}\right) \tilde{v}_{o x}-2 r \tilde{v}_{o r} & =-\mathrm{i} k \frac{\tilde{p}_{o f}}{\Gamma}, \\
\mathrm{i} k\left(U-\frac{c}{\Gamma}\right) \tilde{v}_{o r} & =-\frac{\mathrm{d}_{r} \tilde{p}_{o f}}{\Gamma} .
\end{aligned}
$$

As mentioned in Section 2 (Eq. (44)), in order to achieve a balance between normal stresses in the fluid and the wall material, it is necessary to stipulate that the radial velocity in the outer layer $\tilde{v}_{\text {or }} \sim \delta$. In the outer layer, the radial and tangential velocities are of the same order, and so the tangential velocity $\tilde{v}_{o x} \sim \delta$. The fluid pressure in the outer layer can then be estimated from the outer layer $x$-momentum equation (62). This shows that $\tilde{p}_{\text {of }} \sim O(1)$. For future reference, the magnitudes of the velocity and stress fields in the outer flow are given below:

$$
\tilde{v}_{o x} \sim \delta, \quad \tilde{v}_{o r} \sim \delta, \quad \tilde{\tau}_{o r r} \sim \tilde{p}_{o f} \sim 1, \quad \tilde{\tau}_{w x r} \sim \delta^{3} .
$$

In the above equation, the normal stress in the outer layer $\tilde{\tau}_{\text {orr }}$ is of the same magnitude as the fluid pressure $\tilde{p}_{\text {of }}$ in the outer layer. According to the above estimates, the outer layer velocities and pressure are expanded in an asymptotic series:

$$
\begin{aligned}
& \tilde{v}_{o r}=\delta\left(\tilde{v}_{o r}^{(0)}+\delta \tilde{v}_{o r}^{(1)}+\cdots\right), \\
& \tilde{v}_{o x}=\delta\left(\tilde{v}_{o x}^{(0)}+\delta \tilde{v}_{o x}^{(1)}+\cdots\right), \\
& \tilde{p}_{o f}=\left(\tilde{p}_{o f}^{(0)}+\delta \tilde{p}_{o f}^{(1)}+\cdots\right) .
\end{aligned}
$$

The governing inviscid equations in the outer layer $(61,62)$ and (63) are reduced to a single equation, which, to leading order in $\delta$, takes the following form:

$$
U\left[\mathrm{~d}_{r}^{2}+r^{-1} \mathrm{~d}_{r}-r^{-2}-k^{2}\right] \tilde{v}_{o r}^{(0)}=0 .
$$

This equation is solved for $\tilde{v}_{o r}^{(0)}$, and the solution consistent with the zero radial velocity boundary condition at the centre of the tube $r=0$ is given by:

$$
\tilde{v}_{o r}^{(0)}=A_{1} I_{1}(k r) \text {, }
$$

where $A_{1}$ is a constant that has to be determined from the boundary conditions at the interface. From the continuity equation and the $x$-momentum equation in the outer layer, the expressions for $\tilde{v}_{o r}^{(0)}$ and $\tilde{p}_{o f}^{(0)}$ are evaluated as follows:

$$
\begin{aligned}
& \tilde{v}_{o x}^{(0)}=\mathrm{i} A_{1} I_{0}(k r), \\
& \tilde{p}_{o f}^{(0)}=\left(A_{1} / k\right)\left[-\mathrm{i} k \Gamma_{0}\left(1-r^{2}\right) I_{0}(k r)-2 \Gamma_{0} \mathrm{i} r I_{1}(k r)\right] .
\end{aligned}
$$

Here, $I_{0}$ and $I_{1}$ are modified Bessel functions of the first kind. The equation governing the first correction to the velocity field $\tilde{v}_{o r}^{(1)}$ in the outer layer is given by:

$$
\begin{aligned}
& U\left[\mathrm{~d}_{r}^{2}+r^{-1} \mathrm{~d}_{r}-r^{-2}-k^{2}\right] \tilde{v}_{o r}^{(1)}- \\
& \quad c^{(1)}\left[\mathrm{d}_{r}^{2}+r^{-1} \mathrm{~d}_{r}-r^{-2}-k^{2}\right] \tilde{v}_{o r}^{(0)}=0 .
\end{aligned}
$$

It is easily verified that the solution for the first correction to the velocity field is identical to the leading-order velocity field, and therefore we can set $\tilde{v}_{\text {or }}^{(1)}=0$ without loss of generality. With this, the solution for the first correction to the velocity and the pressure fields in the outer layer are:

$$
\tilde{v}_{o r}^{(1)}=0, \tilde{v}_{o x}^{(1)}=0, \tilde{p}_{o f}^{(1)}=c^{(0)} \tilde{v}_{o x}^{(0)} .
$$

The equations governing the displacement field in the wall medium (13-15) are scaled using the expansions (30), and the leading order governing equations in the wall medium are given by

$$
\begin{aligned}
& \left(\mathrm{d}_{r}+r^{-1}\right) \tilde{u}_{r}^{(0)}+\mathrm{i} k \tilde{u}_{x}^{(0)}=0, \\
& -k^{2}\left(c^{(0)}\right)^{2} \tilde{u}_{x}^{(0)}=-\mathrm{i} k \tilde{p}_{g}^{(0)}+\left(\mathrm{d}_{r}^{2}+r^{-1} \mathrm{~d}_{r}-k^{2}\right) \tilde{u}_{x}^{(0)} \\
& -k^{2}\left(c^{(0)}\right)^{2} \tilde{u}_{r}^{(0)}=-\mathrm{d}_{r} \tilde{p}_{g}^{(0)}+\left(\mathrm{d}_{r}^{2}+r^{-1} \mathrm{~d}_{r}-r^{-2}-k^{2}\right) \tilde{u}_{r}^{(0)} .
\end{aligned}
$$

While deriving the above equations, the ratio of wall to fluid viscosities $\eta_{r}$ is assumed to be an $O(1)$ quantity. It is important to note here that as a consequence of the scalings used in the present work, the inertial stresses in the wall medium are of the same order of magnitude as the elastic stresses in the wall medium. In the earlier analysis of Kumaran [15], however, the scaling assumptions resulted in inertial stresses in the wall being $O(\delta)$ small compared to elastic stresses in the wall medium. As a result, the governing equations in the wall medium in the present analysis contain the wavespeed $c^{(0)}(74)$ and (75), while the governing equations in the wall medium of the previous asymptotic analysis of Kumaran [15] was independent of the wavespeed. The governing equations for the first correction to the displacements are:

$$
\left(\mathrm{d}_{r}+r^{-1}\right) \tilde{u}_{r}^{(1)}+\mathrm{i} k \tilde{u}_{x}^{(1)}=0
$$

$$
\begin{aligned}
-k^{2}\left(c^{(0)}\right)^{2} \tilde{u}_{x}^{(1)}- & 2 k c^{(0)} c^{(1)} \tilde{u}_{x}^{(0)}= \\
& -\mathrm{i} k \tilde{p}_{g}^{(1)}+\left(\mathrm{d}_{r}^{2}+r^{-1} \mathrm{~d}_{r}-k^{2}\right) \tilde{u}_{x}^{(1)}
\end{aligned}
$$

$$
\begin{aligned}
-k^{2}\left(c^{(0)}\right)^{2} & \tilde{u}_{r}^{(1)}-2 k c^{(0)} c^{(1)} \tilde{u}_{r}^{(0)}= \\
& -\mathrm{d}_{r} \tilde{p}_{g}^{(1)}+\left(\mathrm{d}_{r}^{2}+r^{-1} \mathrm{~d}_{r}-r^{-2}-k^{2}\right) \tilde{u}_{r}^{(1)} .
\end{aligned}
$$

It should be noted here that both the leading order and first correction governing equations in the wall medium do not contain the wall to fluid viscosity ratio $\eta_{r}$. The solution to the leading order radial displacement field $\tilde{u}_{r}^{(0)}$ are found by solving $(73,74)$ and $(75)$ :

$$
\tilde{u}_{r}^{(0)}=B_{1} K_{1}(\gamma r)+B_{2} K_{1}(k r)+B_{3} I_{1}(\gamma r)+B_{4} I_{1}(k r)
$$

where $\gamma=k\left[1-\left(c^{(0)}\right)^{2}\right]^{1 / 2}$, and $K_{0}$ and $K_{1}$ are the modified Bessel functions of the second kind. The constants, 
$B_{1}, B_{2}, B_{3}$ and $B_{4}$ are determined from the zero displacement conditions at $r=H$ and from the velocity and stress continuity conditions at the interface. The tangential displacement $\tilde{u}_{x}^{(0)}$ and the pressure in the wall $\tilde{p}_{g}^{(0)}$ are determined respectively from the continuity equation (13) and the $x$-momentum equation (14) in the wall. The equations governing the first correction to the displacement field (76), (77) and (78) are solved for $\tilde{u}_{r}^{(1)}$ :

$\tilde{u}_{r}^{(1)}=B_{1}\left[k^{2} c^{(0)} c^{(1)} r K_{0}(\gamma r) / \gamma\right]-B_{3}\left[k^{2} c^{(0)} c^{(1)} r I_{0}(\gamma r) / \gamma\right]$.

\section{Appendix B}

In this appendix, it is shown that if the displacements in the wall medium are assumed to be $O(\delta)$, then the resulting leading order wavespeed $c^{(0)}$ is always stable as found in the earlier asymptotic analysis of Kumaran [15]. The normal velocity continuity condition $(27)$ indicates that $\tilde{u}_{r}$ at $r=1$ is $O(\delta)$. If $\tilde{u}_{r}$ is assumed to be $O(\delta)$ throughout the wall medium, then $\tilde{u}_{x}$ is also $O(\delta)$ according to the continuity equation (13) in the wall medium. Therefore, the displacement field in the wall is scaled as follows:

$$
\tilde{u}_{r}=\delta\left(\tilde{u}_{r}^{(0)}+\delta \tilde{u}_{r}^{(1)}+\cdots\right), \tilde{u}_{x}=\delta\left(\tilde{u}_{x}^{(0)}+\delta \tilde{u}_{x}^{(1)}+\cdots\right) .
$$

The above expansions are substituted in the boundary conditions at the interface. The normal velocity continuity $\left(\tilde{v}_{r}=-\mathrm{i} k c \tilde{u}_{r}\right)$ becomes:

$$
\delta\left(\tilde{v}_{o r}^{(0)}+\tilde{v}_{w r}^{(0)}\right)=-\mathrm{i} k\left(c^{(0)}+\delta c^{(1)}\right) \delta\left(\tilde{u}_{r}^{(0)}+\delta \tilde{u}_{r}^{(1)}\right) .
$$

To leading order in $\delta$, this yields:

$$
\tilde{v}_{o r}^{(0)}+\tilde{v}_{w r}^{(0)}=-\mathrm{i} k c^{(0)} \tilde{u}_{r}^{(0)} .
$$

The tangential velocity continuity condition $\left(\tilde{v}_{x}-2 \Gamma \tilde{u}_{r}=\right.$ $\left.-\mathrm{i} k c \tilde{u}_{x}\right)$ becomes:

$$
\begin{aligned}
\tilde{v}_{w x}^{(0)}-2 \Gamma_{0} \delta^{-1} & \delta\left(\tilde{u}_{r}^{(0)}+\tilde{u}_{r}^{(1)}+\cdots\right)= \\
& -\mathrm{i} k\left(c^{(0)}+\delta c^{(1)}\right) \delta\left(\tilde{u}_{x}^{(0)}+\tilde{u}_{x}^{(1)}+\cdots\right) .
\end{aligned}
$$

To leading order, this condition yields:

$$
\tilde{v}_{w x}^{(0)}-2 \Gamma_{0} \tilde{u}_{r}^{(0)}=0 .
$$

The tangential stress continuity condition (18) can be similarly scaled, and to leading order, this gives:

$$
\Gamma_{0}\left[-\mathrm{d}_{\xi} \tilde{v}_{w x}^{(0)}\right]=\left(\mathrm{d}_{r} \tilde{u}_{x}^{(0)}+\mathrm{i} k \tilde{u}_{r}^{(0)}\right) .
$$

The normal stress balance (19), on using the above scaling for the displacement field, yields:

$$
-\tilde{p}_{o f}^{(0)}+2 \delta^{2} \Gamma_{0}\left[-\mathrm{d}_{\xi} \tilde{v}_{w r}^{(0)}\right]=\delta\left[-\tilde{p}_{g}^{(0)}+2 \mathrm{~d}_{r} \tilde{u}_{r}^{(0)}\right] .
$$

Here, it should be recalled that, as discussed in Section 2, the pressure in the outer layer in the fluid is $O(1)$. The pressure in the wall medium $\tilde{p}_{g}$ can be estimated from the $x$-momentum equation (14) in the wall, and this shows that $\tilde{p}_{g} \sim \delta$, since $\tilde{u}_{x} \sim \delta$. To leading order in $\delta$, the above equation yields:

$$
\tilde{p}_{o f}^{(0)}=0
$$

Therefore, for this choice scaling of wall displacement quantities, the fluid pressure in the outer layer is zero in the leading approximation, and the fluid pressure in the outer layer is $O(\delta)$. The momentum equations in the outer layer then indicate that $\tilde{v}_{o r}^{(0)}$ and $\tilde{v}_{o x}^{(0)}$ are zero in the leading approximation. On noting the above conclusions regarding the outer layer velocities, the leading order boundary conditions at the interface become:

$$
\begin{aligned}
\tilde{v}_{w r}^{(0)} & =-\mathrm{i} k c^{(0)} \tilde{u}_{r}^{(0)}, \\
\tilde{v}_{w x}^{(0)}-2 \Gamma_{0} \tilde{u}_{r}^{(0)} & =0, \\
\Gamma_{0}\left[-\mathrm{d}_{\xi} \tilde{v}_{w x}^{(0)}\right] & =\left(\mathrm{d}_{r} \tilde{u}_{x}^{(0)}+\mathrm{i} k \tilde{u}_{r}^{(0)}\right) .
\end{aligned}
$$

It is easily verified the equations (89) and (90) are sufficient to determine the leading order wavespeed $c^{(0)}$, since these two equations can be used to eliminate $\tilde{u}_{r}^{(0)}$ to give the following characteristic equation for $c^{(0)}$ :

$$
2 \Gamma_{0} \tilde{v}_{w r}^{(0)}+\mathrm{i} k c^{(0)} \tilde{v}_{w x}^{(0)}=0 .
$$

Here the velocities $\tilde{v}_{w r}^{(0)}$ and $\tilde{v}_{w x}^{(0)}$ have to be evaluated at the wall $(r=1$ or $\xi=0)$. The solutions determined for the wall layer quantities (57) can be used in the above characteristic equation to determine $c^{(0)}$. It can be further verified that, on using the solutions to the wall layer velocities, the above equation (92) reduces to

$$
A i\left(y_{\text {wall }},-1\right)=0 \text {, }
$$

where $y_{\text {wall }}$ denotes that the variable $y$ (53) should be evaluated at the wall i.e. $\xi=0$, which gives $y_{\text {wall }}=(2 \mathrm{i} k)^{1 / 3}\left(-c^{(0)} /\left(2 \Gamma_{0}\right)\right)$. The above characteristic equation (93) is identical to the characteristic equation obtained by Kumaran [15] (see equation 35 in that paper) in the limit $R e^{1 / 3} G /\left(\rho V^{2}\right) \rightarrow 0$. There are multiple solutions to the equation $A i\left(y_{\text {wall }},-1\right)=0$, and all these solutions are negative real quantities (see [15]). Therefore the solutions to $y_{\text {wall }}$ are negative and real, which implies that from the definition $y_{\text {wall }}$, the various solutions to $c^{(0)}$ have negative imaginary parts indicating that the flow is stable for this choice of scalings of the wall displacement field. It is further interesting to note that the leading order wavespeed for this class of 'stable wall modes' (also discussed in [15]) are determined purely from the fluid eigenfunctions, and the wall medium eigenfunctions do not enter at the leading approximation in determining $c^{(0)}$. 


\section{References}

1. P. Krindel, A. Silberberg, J. Colloid Interface Sci. 71, 34 (1979).

2. V. Kumaran, J. Fluid. Mech. 294, 259 (1995).

3. V. Kumaran, J. Fluid. Mech. 302, 117 (1995).

4. L. Srivatsan, V. Kumaran, J. Phys. II France 7, 947 (1997).

5. V. Kumaran, J. Fluid. Mech. 357, 123 (1998).

6. V. Kumaran, J. Fluid. Mech. 362, 1 (1998).

7. V. Kumaran, R. Muralikrishnan, Phys. Rev. Lett. 84, 3310 (2000).

8. V. Kumaran, G.H. Fredrickson, P. Pincus, J. Phys. II France 4, 893 (1994).

9. O.E. Jensen, T.J. Pedley, J. Fluid. Mech. 206, 339 (1989).
10. O.E. Jensen, J. Fluid. Mech. 220, 623 (1990).

11. G. Pedrizzetti, J. Fluid. Mech. 375, 39 (1998).

12. C. Davies, P.W. Carpenter, J. Fluid. Mech. 352, 205 (1997).

13. G.M. Corcos, J.R. Sellars, J. Fluid Mech. 5, 97 (1959).

14. A.E. Gill, J. Fluid Mech. 21, 145 (1965).

15. V. Kumaran, Eur. Phys. J. B 4, 519 (1998).

16. V. Kumaran, J. Fluid. Mech 320, 1 (1996).

17. L. Landau, E. Lifshitz, Theory of Elasticity (Pergamon, New York, 1989)

18. J. Harden, H. Pleiner, P. Pincus, J. Chem. Phys. 94, 5208 (1991).

19. V. Shankar, V. Kumaran, J. Fluid. Mech. 395, 211 (1999).

20. P. Drazin, W. Reid, Hydrodynamic Stability (Cambridge University Press, Cambridge, 1981). 